\title{
Results from EDGES High-Band. III. New Constraints on Parameters of the Early Universe
}

\author{
Raul A. Monsalve ${ }^{1,2,3,4,5}$ (iD), Anastasia Fialkov ${ }^{6,7,8,9,10}$ (D), Judd D. Bowman ${ }^{3}$ (D), Alan E. E. Rogers ${ }^{11}$ (D), Thomas J. Mozdzen ${ }^{3}$ (D), \\ Aviad Cohen ${ }^{12}$, Rennan Barkana ${ }^{12}$, and Nivedita Mahesh ${ }^{3}$ (D) \\ ${ }^{1}$ Department of Physics, McGill University, Montréal, 3600 Rue University, QC H3A 2T8, Canada; Raul.Monsalve@mcgill.ca \\ ${ }^{2}$ McGill Space Institute, McGill University, Montréal, 3550 Rue University, QC H3A 2A7, Canada \\ ${ }^{3}$ School of Earth and Space Exploration, Arizona State University, 781 Terrace Mall, Tempe, AZ 85287, USA \\ ${ }^{4}$ Center for Astrophysics and Space Astronomy, University of Colorado, 2000 Colorado Avenue, Boulder, CO 80309, USA \\ ${ }^{5}$ Facultad de Ingeniería, Universidad Católica de la Santísima Concepción, Alonso de Ribera 2850, Concepción, Chile \\ ${ }^{6}$ Harvard-Smithsonian Center for Astrophysics, Institute for Theory and Computation, 60 Garden Street, Cambridge, MA 02138, USA \\ ${ }^{7}$ Institute of Astronomy, University of Cambridge, Madingley Road, Cambridge CB3 OHA, UK \\ ${ }^{8}$ Kavli Institute for Cosmology, University of Cambridge, Madingley Road, Cambridge CB3 OHA, UK \\ ${ }^{9}$ Department of Physics, The University of Tokyo, 7-3-1 Hongo, Bunkyo, Tokyo 113-0033, Japan \\ ${ }^{10}$ Department of Physics and Astronomy, University of Sussex, Falmer, Brighton BN1 9QH, UK \\ ${ }_{11}^{11}$ Haystack Observatory, Massachusetts Institute of Technology, 99 Millstone Road, Westford, MA 01886, USA \\ ${ }^{12}$ Raymond and Beverly Sackler School of Physics and Astronomy, Tel Aviv University, Tel Aviv 69978, Israel \\ Received 2018 October 9; revised 2019 January 31; accepted 2019 February 14; published 2019 April 16
}

\begin{abstract}
We present new constraints on parameters of cosmic dawn and the epoch of reionization derived from the EDGES High-Band spectrum (90-190 MHz). The parameters are probed by evaluating global $21 \mathrm{~cm}$ signals generated with the recently developed Global $21 \mathrm{~cm}$ tool. This tool uses neural networks trained and tested on $\sim 30,000$ spectra produced with semi-numerical simulations that assume the standard thermal evolution of the cosmic microwave background and the intergalactic medium. From our analysis, we constrain at $68 \%$ (1) the minimum virial circular velocity of starforming halos to $V_{\mathrm{c}}<19.3 \mathrm{~km} \mathrm{~s}^{-1}$, (2) the X-ray heating efficiency of early sources to $f_{\mathrm{X}}>0.0042$, and (3) the lowenergy cutoff of the X-ray spectral energy distribution to $\nu_{\min }<2.3 \mathrm{keV}$. We also constrain the star formation efficiency $\left(f_{*}\right)$, the electron scattering optical depth $\left(\tau_{\mathrm{e}}\right)$, and the mean-free path of ionizing photons $\left(R_{\mathrm{mfp}}\right)$. We recompute the constraints after incorporating into the analysis four estimates for the neutral hydrogen fraction from high- $z$ quasars and galaxies, and a prior on $\tau_{\mathrm{e}}$ from Planck 2018. The largest impact of the external observations is on the parameters that most directly characterize reionization. Specifically, we derive the combined $68 \%$ constraints $\tau_{\mathrm{e}}<0.063$ and $R_{\mathrm{mfp}}>27.5 \mathrm{Mpc}$. The external observations also have a significant effect on $V_{\mathrm{c}}$ due to its degeneracy with $\tau_{\mathrm{e}}$, while the constraints on $f_{*}, f_{\mathrm{X}}$, and $\nu_{\mathrm{min}}$, remain primarily determined by EDGES.
\end{abstract}

Key words: cosmology: observations - early universe - galaxies: high-redshift - methods: data analysis

\section{Introduction}

The sky-averaged, or global, radio spectrum is expected to encode the redshift evolution of the $21 \mathrm{~cm}$ line of neutral hydrogen gas in the intergalactic medium (IGM) during the formation of the first stars and galaxies (Varshalovich \& Khersonskii 1977; Tozzi et al. 2000; Barkana \& Loeb 2001; Furlanetto et al. 2006; Pritchard \& Loeb 2008). At the onset of star formation, stellar Ly $\alpha$ photons couple the spin temperature of the $21 \mathrm{~cm}$ signal to the temperature of the gas, a process that makes the line visible in absorption relative to the radio background radiation (Wouthuysen 1952; Field 1958). The detection by Bowman et al. (2018) of an absorption feature at $\sim 78 \mathrm{MHz}$, with a width of $\sim 20 \mathrm{MHz}$ and an amplitude of $\sim 0.5 \mathrm{~K}$, if confirmed to be of cosmological origin, is the first measurement of the $21 \mathrm{~cm}$ signal from cosmic dawn and would represent direct evidence of the formation of the first stars. The central frequency of the reported feature is in agreement with theoretical predictions and implies efficient star formation in halos of mass below $10^{8} M_{\odot}$ (Mirocha \& Furlanetto 2019). Such a population would manifest itself in future high-redshift galaxy surveys generating a steeper-than-expected UV luminosity function at the faint end (Mirocha et al. 2017; Mirocha \& Furlanetto 2019).

However, the amplitude and shape of the detected radio signal do not comply with standard models of cosmic dawn and reionization, where the absorption is measured against the cosmic microwave background (CMB) and the IGM is heated by X-ray sources after an initial period of adiabatic cooling (e.g., Mesinger et al. 2011; Cohen et al. 2017; Mirocha et al. 2018). The reported absorption amplitude is at least twice as large as predicted, and the observed shape is much flatter at the bottom than expected. These discrepancies have led to many suggestions of exotic physical mechanisms that could produce either a colder IGM temperature at early times (e.g., Tashiro et al. 2014; Muñoz et al. 2015; Barkana 2018; Barkana et al. 2018; Berlin et al. 2018; Fialkov et al. 2018; Hektor et al. 2018; Houston et al. 2018; Muñoz \& Loeb 2018; Sikivie 2019) or a radiation background stronger than the CMB (e.g., Ewall-Wice et al. 2018; Feng \& Holder 2018; Fraser et al. 2018).

Verification of the unexpected EDGES measurement, which was conducted with two "Low-Band" instruments observing in the $50-100 \mathrm{MHz}$ frequency range $(26.4 \gtrsim z \gtrsim 13.2)$, requires independent observations and constraints. Until the EDGES result, the only constraint on the global $21 \mathrm{~cm}$ signal from cosmic dawn in the same frequency range was the upper limit for the absorption amplitude of $0.89 \mathrm{~K}(95 \%)$ established by the LEDA experiment (Bernardi et al. 2016). Previously, the SCI-HI experiment reported upper limits in the range $\sim 1-10 \mathrm{~K}$ on the residual spectral structure after removing a model for the foreground contribution (Voytek et al. 2014). Upper limits on 
the $21 \mathrm{~cm}$ power-spectrum signal have been presented in the range $z=12-18$ by MWA (Ewall-Wice et al. 2016) and $z=20-25$ by LOFAR (Gehlot et al. 2018). An additional, but also currently disputed, potential evidence of new physics at cosmic dawn could come from the measurement of the brightness temperature of the diffuse sky by ARCADE 2, which found a $54 \mathrm{mK}$ "excess" above the CMB at $3.3 \mathrm{GHz}$ (Fixsen et al. 2011). A consistent excess was recently reported using LWA1 data over 40-80 MHz (Dowell \& Taylor 2018). However, the existence of this excess relies on the correct identification and removal of the Galactic and extragalactic contributions. Using a more realistic Galactic model, Subrahmanyan \& Cowsik (2013) showed that the excess could instead correspond to a Galactic contribution that is not accounted for in other analyses.

Compared to cosmic dawn, constraints on the epoch of reionization are tighter and stem from several independent observational probes, none of which has so far reported deviations from traditional astrophysics. The average fraction of neutral hydrogen in the IGM, $\bar{x}_{\mathrm{HI}}$, can be constrained through the Ly $\alpha$ and Ly $\beta$ pixels that are dark in the spectra of high- $z$ quasars. Using this technique and a sample of 22 quasars at $z=5-6$, McGreer et al. (2015) derive the upper limit $\bar{x}_{\mathrm{H}} \leqslant 0.06+0.05(68 \%)$ at $z=5.9$. Neutral hydrogen in the IGM also imprints a "damping wing" absorption feature in the spectrum of high- $z$ quasars. Greig et al. (2017) obtain $\bar{x}_{\mathrm{H}}=0.40_{-0.19}^{+0.21}(68 \%)$ from the damping wing analysis of ULASJ1120+0641, showing that reionization is ongoing at $z=7.08$; while the spectrum of ULASJ1342+0928, the highest-redshift quasar detected so far, yields $\bar{x}_{\mathrm{H} \text { I }}=0.65_{-0.32}^{+0.15}$ $(68 \%)$ at $z=7.54$ in a damping wing analysis by Bañados et al. (2018). The IGM neutral fraction can also be constrained from the emission of $\mathrm{Ly} \alpha$ radiation from Lyman break galaxies (LBGs). In a Bayesian analysis that incorporates reionization simulations and empirical models of the interstellar medium, Mason et al. (2018) determine $\bar{x}_{\mathrm{H} \text { I }}=0.59_{-0.15}^{+0.11}(68 \%)$ at $z=7$ from a sample of LBGs presented in Pentericci et al. (2014). CMB fluctuations provide an independent test of reionization by probing the integrated electron scattering optical depth to recombination, $\tau_{\mathrm{e}}$. Among other values, the Planck satellite recently reported $\tau_{\mathrm{e}}=0.056 \pm 0.007$, which corresponds to a reionization center redshift $z=7.82 \pm 0.71$ assuming a "tanh" phenomenological model (Planck Collaboration VI 2018). For a similar type of model, the data from the EDGES "HighBand" instrument provide independent constraints on the reionization duration $(\Delta z)$ via non-detection of the $21 \mathrm{~cm}$ line in the 90-190 MHz range (Monsalve et al. 2017b): at $\geqslant 2 \sigma$ significance, the data rule out models with $\Delta z<1$ at $z \sim 8.5$ and higher than $\Delta z=0.4$ across most of the $14.8 \geqslant z \geqslant 6.5$ range. These constraints implicitly correspond to reionization scenarios where the $21 \mathrm{~cm}$ spin temperature of neutral hydrogen is saturated, i.e., much higher than the microwave background, due to prior IGM heating. Monsalve et al. (2017b) also explored and ruled out $21 \mathrm{~cm}$ models that take the opposite extreme assumption, i.e., total Ly $\alpha$ coupling but no IGM heating before reionization. In this case, the hydrogen neutral fraction was also modeled using the "tanh" expression. As a reference result, Monsalve et al. (2017b) ruled out at $\geqslant 2 \sigma$ all the reionization models with total $\operatorname{Ly} \alpha$ coupling but no IGM heating that produce $\bar{x}_{\mathrm{H}} \leqslant 1 \%$ at $z=6$ and have an optical depth in the range $0.086 \geqslant \tau_{\mathrm{e}} \geqslant 0.038$. Finally, there are also constraints on the $21 \mathrm{~cm}$ power-spectrum signal at $z=8.6$ from GMRT (Paciga et al. 2013) and at $z=7.1$ from MWA (Beardsley et al. 2016), while the tightest upper limits have been reported by LOFAR in the range $z=9.6-10.6$ (Patil et al. 2017). ${ }^{13}$

Recently, global radio spectra were analyzed using astrophysical models for the first time (Singh et al. 2017, 2018; Monsalve et al. 2018). A set of 193 models from a parameter study by Cohen et al. (2017) was evaluated using data from the SARAS 2 experiment in the $110-200 \mathrm{MHz}$ band, which allowed to rule out, at $>5 \sigma$ significance, 25 models that share inefficient X-ray heating and rapid reionization (Singh et al. 2017, 2018). A much broader study was done using EDGES High-Band data (Monsalve et al. 2018, M18 hereafter), which evaluated 10,000 models generated with the $21 \mathrm{cmFAST}$ code (Mesinger \& Furlanetto 2007; Mesinger et al. 2011). M18 constrained the following parameters of cosmic dawn and reionization: the minimum virial temperature $\left(T_{\mathrm{vir}}^{\mathrm{min}}\right)$ and ionizing efficiency $(\zeta)$ of star-forming halos, as well as the integrated soft-band X-ray luminosity $\left(L_{\mathrm{X}<2 \mathrm{keV}} / \mathrm{SFR}\right)$ and threshold energy for self-absorption $\left(E_{0}\right)$ of the first galaxies. For reference, using EDGES data alone, M18 disfavored $(68 \%) \log _{10}\left(T_{\mathrm{vir}}^{\min } / \mathrm{K}\right)>5.5$ and $\zeta>154.6$, as well as the intermediate range of X-ray luminosity $38.8<$ $\log _{10}\left(L_{\mathrm{X}<2 \mathrm{keV}} / \mathrm{SFR} / \mathrm{erg} \mathrm{yr} \mathrm{s}^{-1} M_{\odot}^{-1}\right)<40.4$. Further, combining (1) the EDGES High-Band data, (2) an estimate for $\tau_{\mathrm{e}}$ from Planck 2016, and (3) constraints on $\bar{x}_{\mathrm{H}}$ from quasars at $z=5.9$ and $z=7.08$ resulted in significantly stronger constraints on $\zeta$ and $T_{\text {vir }}^{\min }$, with EDGES contributing to produce slightly better results than those derived in Greig \& Mesinger (2017a) using only the information on $\tau_{\mathrm{e}}$ and $\bar{x}_{\mathrm{H} \mathrm{I}}$.

In this paper we use the EDGES High-Band data to evaluate a different set of astrophysical models, which were generated with the Global21 cm global signal emulator described in detail by A. Cohen et al. (2019, in preparation). This tool is based on neural networks trained on 29,641 outputs of seminumerical simulations of cosmic dawn and reionization described in detail in Visbal et al. (2012), Fialkov \& Barkana (2014), and Cohen et al. (2017). The simulations make standard assumptions for the temperatures of the CMB and the IGM, not taking into account the exotic physics invoked to explain the EDGES Low-Band result. To produce the simulations, seven astrophysical parameters were varied in the widest possible range: the minimum virial circular velocity of star-forming halos $\left(V_{\mathrm{c}}\right)$, the star formation efficiency (SFE) $\left(f_{*}\right)$, the X-ray heating efficiency of early sources $\left(f_{\mathrm{X}}\right)$, the lowenergy cutoff $\left(\nu_{\mathrm{min}}\right)$ of the X-ray spectral energy distribution (SED), the slope $(\alpha)$ of the X-ray SED, the mean-free path of ionizing photons $\left(R_{\mathrm{mfp}}\right)$, and $\tau_{\mathrm{e}}$. Global21 cm interpolates between the outputs of the semi-numerical simulations and produces global signals for any combination of parameters.

Here, we generate 6.4 million global signals using $\mathrm{Globa} 121 \mathrm{~cm}$ and conduct a Bayesian analysis that rigorously maps the posterior probability density function (PDF) of six of the astrophysical parameters: $V_{\mathrm{c}}, f_{*}, f_{\mathrm{X}}, \nu_{\mathrm{min}}, R_{\mathrm{mfp}}$, and $\tau_{\mathrm{e}}$. We fix the value of $\alpha$ as it only mildly affects the results. We derive constraints on the parameters first using the EDGES High-Band data alone, and then combining them with a prior on $\tau_{\mathrm{e}}$ from Planck and constraints on $\bar{x}_{\mathrm{H}}$ from high- $z$ quasars and galaxies. Our main results are the 1D and 2D PDFs of each parameter and parameter pair, obtained after marginalizing over

\footnotetext{
${ }^{13}$ The PAPER $21 \mathrm{~cm}$ power-spectrum constraint at $z=8.4$ of Ali et al. (2015) has been retracted in Ali et al. (2018).
} 
the rest of the astrophysical parameters as well as the parameters that account for the contribution of diffuse foregrounds to the radio spectrum.

In addition to exploring more parameters, six instead of four, we improve on M18 by including in our combined analysis an updated prior on $\tau_{\mathrm{e}}$ (Planck 2018 instead of 2016), a constraint on $\bar{x}_{\mathrm{H} \text { I }}$ at $z=7.54$ by Bañados et al. (2018) from the ULASJ1342+0928 quasar, and a constraint on $\bar{x}_{\mathrm{H} \text { I }}$ at $z=7$ by Mason et al. (2018) from LBGs. In general, the parameters we explore are different from those in M18, and although some of them overlap, here we explore them over a wider range. This makes it difficult to compare in detail our results with those of M18. On the other hand, it enables us to derive independent conclusions about the astrophysics of the early universe. As in M18, we do not incorporate the EDGES Low-Band spectrum into our analysis, saving that for future work.

The paper is organized as follows. In Section 2 we briefly describe the $21 \mathrm{~cm}$ astrophysical parameters. In Section 3 we detail our analysis procedure. In Section 4 we present the results obtained from the analysis of EDGES data alone, the external constraints alone, and their combination. In Section 5 we discuss the results and compare them with those for models from $21 \mathrm{cmFAST}$. Finally, in Section 6 we summarize this work.

\section{Astrophysical Parameters}

The Global $21 \mathrm{~cm}$ code outputs a global $21 \mathrm{~cm}$ signal over the redshift range $6<z<50$ given a combination of key astrophysical parameters in less than one second of computing time. The code employs neural networks that were trained on 29,641 global spectra produced with a hybrid simulation of the high-redshift universe (Visbal et al. 2012; Fialkov \& Barkana 2014; Cohen et al. 2017). For an input set of astrophysical parameters, the simulation generates a realization of the $21 \mathrm{~cm}$ signal within large cosmological volumes $\left(384^{3}\right.$ comoving $\left.\mathrm{Mpc}^{3}\right)$ and over a wide redshift range $(z=6-60)$. The global spectra are obtained by averaging the 3D $21 \mathrm{~cm}$ fields over the box at every redshift. Each simulation takes $\sim 4$ hr to run on a desktop, and the ensemble of 29,641 models was produced using the Odyssey cluster at Harvard University. ${ }^{14}$ All these runs were executed with the same set of initial conditions for large-scale density and velocity fields at $z=60$, and assume $\Lambda \mathrm{CDM}$ with the standard cosmological parameters (Planck Collaboration XVI 2014). The simulation follows the hierarchical growth of structure, tracks star formation averaged over scales of $\sim 3 \mathrm{Mpc}$, and follows the evolution of inhomogeneous Ly $\alpha$, Lyman-Werner (11.2-13.6 eV), X-ray, and ionizing radiative backgrounds. The simulation takes into account the effect of relative streaming velocity (Visbal et al. 2012), Lyman-Werner radiation (Fialkov et al. 2013), and photoheating feedback on star formation (Cohen et al. 2016).

In the simulation, the high-redshift astrophysics is parameterized with seven parameters: $V_{\mathrm{c}}, f_{*}, f_{\mathrm{X}}, \nu_{\mathrm{min}}, \alpha, R_{\mathrm{mfp}}$, and $\zeta$. The Global2 $1 \mathrm{~cm}$ code receives as input the same parameters except for $\zeta$; the code uses instead the CMB optical depth, which is related to $\zeta$ by a one-to-one mapping. In the rest of this section we briefly describe the parameters and their impact on the global signal. For detailed descriptions we point the reader to Cohen et al. (2017) and A. Cohen et al. (2019, in preparation). An example is given in Figure 1, where

\footnotetext{
${ }^{14}$ https://www.rc.fas.harvard.edu/odyssey/
}
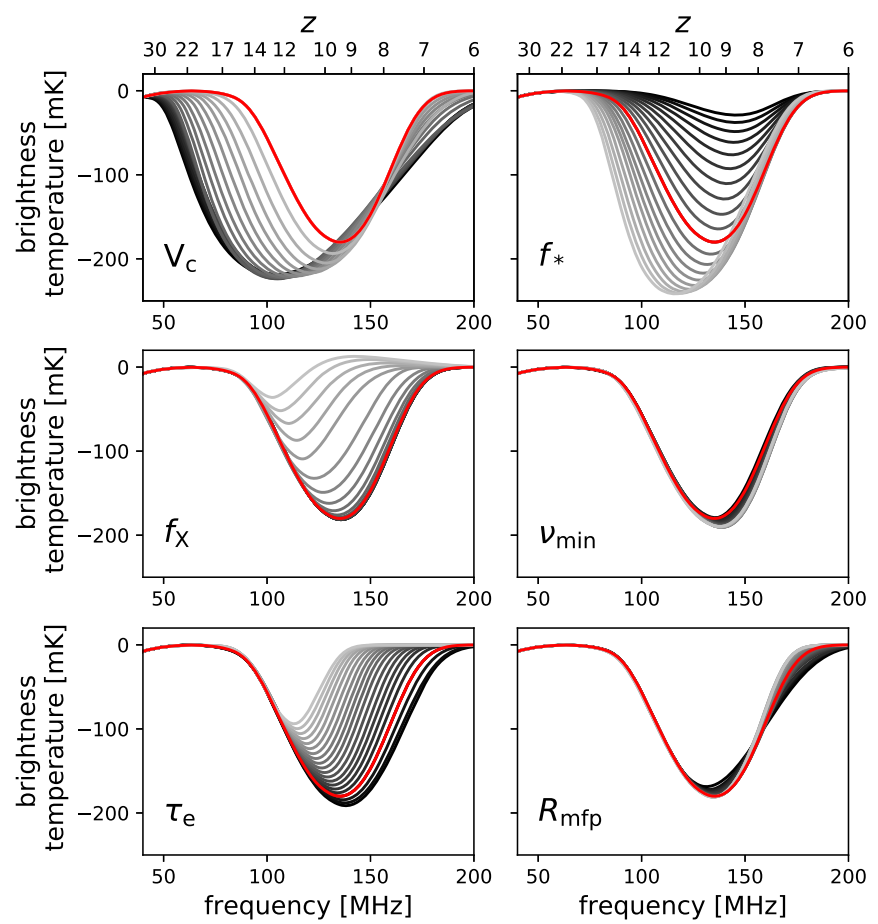

Figure 1. Example of dependence of the global $21 \mathrm{~cm}$ signal on the parameters constrained. The signals are produced using the $\mathrm{Global} 21 \mathrm{~cm}$ code (A. Cohen et al. 2019, in preparation). Each panel shows variations in one parameter relative to a common reference. The colors of the signals, from black to light gray, represent the parameter value going from lowest to highest, spanning the ranges described in Section 2 and listed in Table 1 . For $V_{\mathrm{c}}, f_{*}$, and $f_{\mathrm{X}}$, the sampling is done evenly in $\log _{10}$ scale. The red signal is the common reference, with parameters $V_{\mathrm{c}}=76.5 \mathrm{~km} \mathrm{~s}^{-1}, f_{*}=0.026, f_{\mathrm{X}}=3.4 \times 10^{-3}$, $\nu_{\min }=0.25 \mathrm{keV}, \tau_{\mathrm{e}}=0.06$, and $R_{\mathrm{mfp}}=31 \mathrm{Mpc}$.

Table 1

Parameter Ranges and Sampling Scale

\begin{tabular}{lcccc}
\hline \hline Parameter & Min & Max & Unit & Scale \\
\hline$V_{\mathrm{c}}$ & 4.2 & 76.5 & $\mathrm{~km} \mathrm{~s}^{-1}$ & $\log _{10}$ \\
$f_{*}$ & $10^{-3}$ & 0.5 & $\ldots$ & $\log _{10}$ \\
$f_{\mathrm{X}}$ & $10^{-5}$ & 10 & $\ldots$ & $\log _{10}$ \\
$\nu_{\min }$ & 0.1 & 3 & $\mathrm{keV}$ & linear \\
$\tau_{\mathrm{e}}$ & 0.055 & 0.09 & $\ldots$ & linear \\
$R_{\mathrm{mfp}}$ & 10 & 50 & $\mathrm{Mpc}$ & linear \\
\hline
\end{tabular}

we show the effect of changes in all the parameters except $\alpha$, which is kept fixed at $\alpha=-1.3$. In Table 1 we list the parameter ranges explored in this paper, as well as the scale used to sample these ranges with Global2 $1 \mathrm{~cm}$. Although our parameterization intends to characterize the large-scale physics of cosmic dawn and reionization, it is not necessarily optimized for the global $21 \mathrm{~cm}$ signal. Therefore, the effect of some of the parameters on the global signal is degenerate. We discuss this point in Section 5.1.

Minimum virial circular velocity. In the hierarchical picture of structure formation, low-mass halos form at higher redshifts and are more numerous than high-mass halos. Therefore, in the cases with lower threshold mass for star formation, $M_{\min }$, stars form earlier, leading to an earlier onset of the $\operatorname{Ly} \alpha$ coupling and shifting the descending slope of the $21 \mathrm{~cm}$ absorption feature to lower frequencies. In Global21 cm we use the minimum 
virial circular velocity,

$$
V_{\mathrm{c}} \sim 16.9\left(\frac{M_{\min }}{10^{8}}\right)^{1 / 3}\left(\frac{1+z}{10}\right)^{1 / 2} \mathrm{~km} \mathrm{~s}^{-1},
$$

instead of $M_{\min }$ because $V_{\mathrm{c}}$ is less strongly dependent on redshift (Barkana 2016).

$V_{\mathrm{c}}$ is primarily set by the cooling channel. Molecular hydrogen cooling fuels star formation in halos with $V_{\mathrm{c}}=4.2-16.5 \mathrm{~km} \mathrm{~s}^{-1}$, which corresponds to halo masses $M_{\mathrm{h}} \sim 1 \times 10^{6}-8 \times 10^{7} M_{\odot}$ at $z=10$, while atomic hydrogen cooling occurs for $V_{\mathrm{c}}>16.5 \mathrm{~km} \mathrm{~s}^{-1}$ (Barkana 2016). In addition to radiative cooling, star formation is affected by numerous feedback processes that result in spatial variation of $V_{\mathrm{c}}$ as well as in its dependence on background radiation fields (e.g., Machacek et al. 2001; Fialkov et al. 2012, 2013). To probe different cooling and feedback mechanisms, in this paper we vary $V_{\mathrm{c}}$ in a broad range, from $4.2 \mathrm{~km} \mathrm{~s}^{-1}$, corresponding to the minimum value for molecular hydrogen cooling, to $76.5 \mathrm{~km} \mathrm{~s}^{-1}$, representing atomic hydrogen cooling and inefficient star formation in smaller halos due to, e.g., supernovae feedback.

Star formation efficiency. The SFE corresponds to the fraction of gas in dark matter halos that is converted into stars. Higher values of SFE result in an earlier onset of Ly $\alpha$ coupling, as well as in a faster build-up of X-ray and ionizing radiation backgrounds. The SFE depends on feedback mechanisms, the metalicity of the gas, and the halo mass. Star formation simulations in metal-poor environments show large scatter, with an SFE between $\sim 0.1 \%$ and $\sim 10 \%$ in halos of $M_{\mathrm{h}} \sim 10^{8}-10^{10} M_{\odot}$ at $z \sim 10$ (e.g., $\mathrm{Xu}$ et al. 2016; Ceverino et al. 2017; Ma et al. 2018). Abundance matching techniques applied to $z \geqslant 6$ galaxies find that the SFE peaks at $\sim 30 \%$ for halos of $M_{\mathrm{h}} \sim 10^{11}-10^{12} M_{\odot}$, dropping to $\sim 10 \%$ at $M_{\mathrm{h}} \sim 10^{10} M_{\odot}$ and $M_{\mathrm{h}} \sim 10^{13} M_{\odot}$ (Behroozi \& Silk 2015; Mason et al. 2015; Mashian et al. 2016; Sun \& Furlanetto 2016; Mirocha et al. 2017). Finally, observations of dwarf galaxies infer an SFE $\sim 0.01 \%-0.1 \%$ at $z \sim 10$ (Read et al. 2016).

We use the following SFE- $M_{\mathrm{h}}$ dependence for the models in this study (Cohen et al. 2017):

$$
\operatorname{SFE}\left(M_{\mathrm{h}}\right)= \begin{cases}f_{*} & M_{\text {atomic }}<M_{\mathrm{h}}, \\ f_{*} \frac{\log \left(M_{\mathrm{h}} / M_{\min }\right)}{\log \left(M_{\text {atomic }} / M_{\min }\right)} & M_{\text {min }}<M_{\mathrm{h}}<M_{\text {atomic }}, \\ 0 & M_{\mathrm{h}}<M_{\min },\end{cases}
$$

where $M_{\min }$ is the minimum cooling mass of star-forming halos, $M_{\text {atomic }}$ is the minimum halo mass for atomic cooling, and $f_{*}$ is a parameter that stands for the SFE at the high-mass end. We vary $f_{*}$ over the wide range $0.1 \%-50 \%$.

X-ray SED low-frequency cutoff. The process of IGM X-ray heating can be characterized in terms of the shape of the X-ray SED and the total luminosity. The IGM heating rate, and thus the evolution of the gas temperature, depends on the amount of energy injected by X-ray sources below $\sim 2 \mathrm{keV}$. Dust in host galaxies prevents soft X-rays below $\mathcal{O}(0.1) \mathrm{keV}$ from penetrating the IGM, imposing a low-energy cutoff in the spectrum of the injected photons (e.g., Das et al. 2017). On the other hand, hard X-rays are barely absorbed; they free-stream and add up to form an X-ray background.
In Global $21 \mathrm{~cm}$ we model the X-ray SED as a power law with slope $\alpha$ and low-energy cutoff $\nu_{\text {min }}$. However, we find that variations in $\alpha$ lead to very weak variations of the global signal relative to the sensitivity of the EDGES High-Band data. Therefore, here we fix the value of $\alpha$ at -1.3 . Higher values of $\nu_{\text {min }}$ lead to the effective hardening of the X-ray SED, less efficient heating, and, as a result, deeper $21 \mathrm{~cm}$ absorption with a higher central frequency. This scenario resembles the effect of X-ray binaries (XRBs), one of the most plausible sources to dominate high-redshift X-ray emission. XRBs are expected to have a hard X-ray SED that peaks at $\sim 1-3 \mathrm{keV}$ and has a highenergy tail following a power law with slope $\alpha \sim-1.5$ (Mirabel et al. 2011; Fragos et al. 2013). We vary $\nu_{\min }$ in the range $0.1-3 \mathrm{keV}$, which is wide enough to explore the effects of host galaxy absorption as well as hard X-rays.

X-ray efficiency. The total X-ray luminosity of early sources satisfies the following relation, derived from observations of nearby starburst galaxies and XRBs (Grimm et al. 2003; Gilfanov et al. 2004; Mineo et al. 2012):

$$
\frac{L_{\mathrm{X}}}{\mathrm{SFR}}=3 \times 10^{40} f_{\mathrm{X}} \operatorname{erg~s}^{-1} M_{\odot}^{-1} \mathrm{yr}
$$

where $L_{\mathrm{X}}$ is the total $\mathrm{X}$-ray luminosity emitted in the range $\nu_{\text {min }}-95 \mathrm{keV}$, SFR is the star formation rate (which in our parameterization is a function of $M_{\mathrm{h}}, f_{*}$, and $z$, as well as of the large-scale overdensity and relative velocity between dark matter and gas), and $f_{\mathrm{X}}$ is the $\mathrm{X}$-ray efficiency of sources, which is our parameter in Global21 cm. Fialkov et al. (2017) found that the unresolved soft X-ray background measured by the Chandra X-ray observatory (Lehmer et al. 2012) imposes an upper limit on $f_{\mathrm{X}}$ in the range $\sim 10-500$, depending on the nature of the X-ray sources, the halo cooling channel, and the reionization history. For high values of $f_{\mathrm{X}}$, the contribution of $\mathrm{X}$-rays to reionization becomes significant (up to $\sim 50 \%$ in the case with $f_{\mathrm{X}}=422$ and $\nu_{\min }=0.2 \mathrm{keV}$, Fialkov et al. 2017), and the absorption trough is shallow and occurs at low frequencies. Low values of $f_{\mathrm{X}}$ result in deep absorption troughs centered at high frequencies. This has made it possible to exclude models with low $f_{\mathrm{X}}$ (for some values of $V_{\mathrm{c}}$ and $f_{*}$ ) using SARAS 2 data (Singh et al. 2017, 2018). Here we vary $f_{\mathrm{X}}$ over the wide range $10^{-5}-10$.

Mean-free path of ionizing photons. During reionization, the distance to which ionizing photons can propagate into the IGM determines the physical size of ionized regions. This distance depends on the abundance, density, and structure of photon sinks-absorption systems such as Lyman limit systems, and the corresponding recombinations of these systems. In our parameterization we explore the mean-free path of ionizing photons, $R_{\mathrm{mfp}}$, which we vary over $10-50 \mathrm{Mpc}$ (Alvarez \& Abel 2012; Greig \& Mesinger 2017b). The effect of this parameter is only manifested after the onset of reionization. Higher values of $R_{\mathrm{mfp}}$ lead to a faster reionization and a steeper $21 \mathrm{~cm}$ signal at the high-frequency end, which can be constrained by EDGES High-Band.

Electron scattering optical depth. The last independent parameter is the ionizing efficiency of sources, $\zeta$ (Greig \& Mesinger 2017a, M18). However, because CMB experiments probe the CMB optical depth instead of $\zeta$, Global21 cm was constructed to receive $\tau_{\mathrm{e}}$ instead of $\zeta$ as an input parameter. The CMB optical depth measures the total column density of ionized gas, and is thus a function of the reionization history, $\bar{x}_{\mathrm{H}}$, which 
is inferred from the simulations and depends on all the astrophysical parameters. Given the evolution of $\bar{x}_{\mathrm{H}}$ with redshift and for a mass-independent ionizing efficiency, we find a one-toone relation between $\zeta$ and $\tau_{\mathrm{e}}$. The mapping between these two parameters is done using a neural network that was trained on a set of 27,455 cases and tested with 2186 cases (A. Cohen et al. 2019 , in preparation). Increasing $\tau_{\mathrm{e}}$ while keeping the other parameters fixed amounts to a higher $\zeta$ and a faster depletion of neutral gas. This results in an earlier reionization and a shallower $21 \mathrm{~cm}$ absorption with the trough shifted to lower frequencies, as well as a reduced emission feature if such exists. Sixty-eight percent confidence constraints from the Planck 2016 release include $\tau_{\mathrm{e}}=0.066 \pm 0.016,0.078 \pm 0.019$ (Planck Collaboration XIII 2016), $0.055 \pm 0.009$ (Planck Collaboration XLVI 2016), and $0.058 \pm 0.012$ (Planck Collaboration XLVII 2016). Considering these estimates, we explore the range $\tau_{\mathrm{e}}=$ $0.055-0.09$. The lower limit of the range, $\tau_{\mathrm{e}}=0.055$, was determined from the upper limit on $\bar{x}_{\mathrm{H}}$ reported by McGreer et al. (2015), of $\bar{x}_{\mathrm{H}} \leqslant 0.06+0.05(68 \%)$ at $z=5.9$. For our Global $21 \mathrm{~cm}$ models, this upper limit results in a $<1 \%$ probability for $\tau_{\mathrm{e}}<0.056$ when considering the range $\tau_{\mathrm{e}}=0.055-0.09$ (see Section 4.3). In Section 4.2 we discuss how new 2018 results from high- $z$ galaxies (Mason et al. 2018) and Planck (Planck Collaboration VI 2018) warrant extending the range to lower values of $\tau_{\mathrm{e}}$ in future studies. The upper limit of our range, $\tau_{\mathrm{e}}=0.09$, is high considering current constraints. However, exploring a wide range is useful because $\tau_{\mathrm{e}}$ is modeldependent and our $\bar{x}_{\mathrm{H} \text { I }}$ models do not correspond to those that were used by Planck.

\section{Analysis}

Exploring rigorously the parameter space of six dimensions described in Section 2 with high resolution is computationally expensive. Considering the low sensitivity of the global $21 \mathrm{~cm}$ signal to changes in $\nu_{\min }$ and $R_{\mathrm{mfp}}$, we explore the six parameters by dividing the space into two subsets of five parameters each. In one subset, the fifth parameter is $\nu_{\min }$ and $R_{\text {mfp }}$ is fixed at $30 \mathrm{Mpc}$. In the other subset, the fifth parameter is $R_{\mathrm{mfp}}$ and $\nu_{\min }$ is fixed at $0.5 \mathrm{keV}$. We generate the $21 \mathrm{~cm}$ signals by evaluating the $\mathrm{Global} 21 \mathrm{~cm}$ code at 20 values per parameter over a regular grid in the ranges described in Section 2 and Table 1 . This produces a total of $20^{5}=3.2$ million models for each five-parameter set. Because of their large dynamic ranges, the sampling for $V_{\mathrm{c}}, f_{*}$, and $f_{\mathrm{X}}$ is done in $\log _{10}$ scale, while $\tau_{\mathrm{e}}, \nu_{\mathrm{min}}$, and $R_{\mathrm{mfp}}$ are sampled in linear scale.

Following M18, we constrain the parameters by computing their marginalized posterior PDFs within a Bayesian framework. We first derive constraints using EDGES data alone, and then incorporating into the analysis external estimates for $\tau_{\mathrm{e}}$ and $\bar{x}_{\mathrm{H} \text { I }}$. We describe the analyses next.

\subsection{EDGES-only Analysis}

In the EDGES-only analysis we start by fitting our model for the diffuse foregrounds to the difference $d-m_{21}\left(\boldsymbol{\theta}_{21}\right)$, where $d$ is the spectrum measured by EDGES in the range $90-190 \mathrm{MHz}$ (Monsalve et al. 2017b) and $m_{21}\left(\boldsymbol{\theta}_{21}\right)$ represents each $21 \mathrm{~cm}$ signal that is produced by evaluating Global2 $1 \mathrm{~cm}$ at the vector of $21 \mathrm{~cm}$ astrophysical parameters $\boldsymbol{\theta}_{21}$. The diffuse foreground model is given by (Mozden et al. 2016; Monsalve et al. 2017a, 2017b, M18)

$$
m_{\mathrm{fg}}\left(\boldsymbol{\theta}_{\mathrm{fg}}\right)=\sum_{i=0}^{N_{\mathrm{fg}}-1} a_{i} \nu^{-2.5+i}=A \boldsymbol{\theta}_{\mathrm{fg}},
$$

where $\nu$ is frequency, $N_{\mathrm{fg}}=5$ is the number of foreground terms needed to fit the spectrum over $100 \mathrm{MHz}$ (Monsalve et al. $2017 \mathrm{~b}, \mathrm{M} 18), A$ is a matrix with columns corresponding to the $\nu^{-2.5+i}$ basis functions, and $\boldsymbol{\theta}_{\mathrm{fg}}$ is the vector of foreground polynomial coefficients with elements $a_{i}$.

We fit Equation (4) to $d-m_{21}\left(\boldsymbol{\theta}_{21}\right)$ using least squares. The best-fit foreground parameters and model are denoted as $\hat{\boldsymbol{\theta}}_{\mathrm{fg}}$ and $\hat{m}_{\mathrm{fg}}$, respectively. The uncertainty of $\hat{\boldsymbol{\theta}}_{\mathrm{fg}}$ is encapsulated in their $5 \times 5$ covariance matrix, $C=\left(A^{T} \Sigma^{-1} A\right)^{-1}$, where $\Sigma$ is the $N_{\nu} \times N_{\nu}$ covariance matrix of the measured spectrum and $N_{\nu}$ is the number of spectral channels. We construct $\Sigma$ as a diagonal matrix where each element on the diagonal is the sum of the channel variance due to thermal noise and systematic uncertainty. For our channel width of $390.6 \mathrm{kHz}$, the standard deviation of the thermal noise is $\approx 40,6$, and $3 \mathrm{mK}$ at 90,140 , and $190 \mathrm{MHz}$, respectively. The systematic uncertainty estimate has a standard deviation of $35 \mathrm{mK}$ (M18). Finally, the $N_{\nu} \times N_{\nu}$ covariance matrix of $\hat{m}_{\mathrm{fg}}$ is given by $\Sigma_{\mathrm{fg}}=A C A^{T}$.

With the definitions above, and as derived in M18, the likelihood of the data as a function of $\boldsymbol{\theta}_{21}$ after marginalizing over the uncertainty of $\hat{\boldsymbol{\theta}}_{\mathrm{fg}}$ is given by

$$
\begin{aligned}
& \mathcal{L}\left(d \mid \boldsymbol{\theta}_{21}\right)=\sqrt{\frac{(2 \pi)^{N_{\mathrm{fg}}-N_{\nu}}}{|\Sigma|\left|C^{-1}\right|}} \exp \left\{-\frac{1}{2}\left[d-m_{21}\left(\boldsymbol{\theta}_{21}\right)-\hat{m}_{\mathrm{fg}}\right]^{T}\right. \\
& \left.\times(\Sigma+V)^{-1}\left[d-m_{21}\left(\boldsymbol{\theta}_{21}\right)-\hat{m}_{\mathrm{fg}}\right]\right\},
\end{aligned}
$$

where $V=\left(\Sigma_{\mathrm{fg}}^{-1}-\Sigma^{-1}\right)^{-1}$. This likelihood is evaluated for each of the 6.4 million global signals produced with Global2 $1 \mathrm{~cm}$. Defining the prior distribution of the $\boldsymbol{\theta}_{21}$ parameters as $\mathcal{P}\left(\boldsymbol{\theta}_{21}\right)$, the $1 \mathrm{D}$ and $2 \mathrm{D}$ posterior PDFs are obtained by numerically integrating the product $\mathcal{L}\left(d \mid \boldsymbol{\theta}_{21}\right) \mathcal{P}\left(\boldsymbol{\theta}_{21}\right)$ over the $\boldsymbol{\theta}_{21}$ parameters being marginalized. In the EDGESonly analysis we assume a uniform prior distribution for all the parameters over the ranges listed in Table 1 (uniform in $\log _{10}$ for $V_{\mathrm{c}}, f_{*}$, and $f_{\mathrm{X}}$, and in linear scale for the others).

\subsection{Combined Analysis}

Following Greig \& Mesinger (2017a) and M18, and improving over M18 by using a more recent prior on $\tau_{\mathrm{e}}$ and two additional constraints on $\bar{x}_{\mathrm{H}}$, we derive constraints on the high $z$ astrophysical parameters after incorporating into our analysis the following external estimates:

1. $\tau_{\mathrm{e}}$ estimate from Planck: We use as a prior the estimate $\tau_{\mathrm{e}}=0.056 \pm 0.007(68 \%)$ from Planck Collaboration VI (2018), which we model as Gaussian. Planck Collaboration VI (2018) report several values, including the baseline result $\tau_{\mathrm{e}}=0.054 \pm 0.007$ derived from the analysis that considers the Planck CMB power spectra and lensing reconstruction. Here we choose to use $\tau_{\mathrm{e}}=0.056 \pm 0.007$, obtained from the analysis that also incorporates baryon acoustic oscillation measurements.

2. $\bar{x}_{\mathrm{H} \text { I }}$ constraint at $z=5.9$ : We use the upper limit on $\bar{x}_{\mathrm{H}}$ from McGreer et al. (2015), derived from the fraction of 
pixels that are dark in the $\operatorname{Ly} \alpha$ and $\operatorname{Ly} \beta$ regions of high- $z$ quasar spectra (Mesinger 2010). We model this upper limit as a flat probability for $\bar{x}_{\mathrm{H}} \leqslant 0.06$ and a decreasing probability for $\bar{x}_{\mathrm{H}}>0.06$, which follows a Gaussian with center $\bar{x}_{\mathrm{H}}=0.06$ and width $\sigma=0.05$.

3. $\bar{x}_{\mathrm{H}}$ estimate at $z=7.08$ : We use the $\bar{x}_{\mathrm{H}}$ PDF estimated by Greig et al. (2017) from the Ly $\alpha$ damping wing analysis of the ULASJ1120+0641 quasar (Mortlock et al. 2011). Specifically, we use their result for the "Small H II" reionization morphology (Mesinger et al. 2016). From this PDF, the $68 \%$ estimate is $\bar{x}_{\mathrm{HI}}=0.40_{-0.19}^{+0.21}$.

4. $\bar{x}_{\mathrm{H}}$ estimate at $z=7.54$ : We use the most conservative (i.e., widest) $\bar{x}_{\mathrm{H}}$ PDF estimated by Bañados et al. (2018) from the Ly $\alpha$ damping wing analysis of the ULASJ1342 +0928 quasar. This estimate accounts for uncertainty in the quasar's intrinsic emission through numerical simulations normalized to the average continuum emission of analog quasars in the Sloan Digital Sky Survey quasar catalog (Pâris et al. 2017). From this PDF, the $68 \%$ estimate is $\bar{x}_{\mathrm{H} \text { I }}=0.65_{-0.32}^{+0.15}$.

5. $\bar{x}_{\mathrm{H} \text { I }}$ estimate at $z=7:$ We use the $\bar{x}_{\mathrm{H} \text { I }}$ PDF computed by Mason et al. (2018) in their analysis of Ly $\alpha$ transmission from the 68 LBGs reported by Pentericci et al. (2014). Their analysis incorporates reionization simulations and empirical models of radiative transfer effects in the interstellar medium, yielding the $68 \%$ estimate $\bar{x}_{\mathrm{HI}}=0.59_{-0.15}^{+0.11}$.

The PDFs corresponding to these constraints are depicted in Figure 2. We consider the Planck $\tau_{\mathrm{e}} \mathrm{PDF}$ a prior because it corresponds to a parameter directly explored in our analysis. This PDF enters into our analysis through $\mathcal{P}\left(\tau_{\mathrm{e}}\right)$. The other constraints are incorporated through an additional likelihood factor, $\mathcal{L}\left(\bar{x}_{\mathrm{H}} \mid \boldsymbol{\theta}_{21}\right)$, that multiplies the product $\mathcal{L}\left(d \mid \boldsymbol{\theta}_{21}\right) \mathcal{P}\left(\boldsymbol{\theta}_{21}\right)$. $\mathcal{L}\left(\bar{x}_{\mathrm{H}} \mid \boldsymbol{\theta}_{21}\right)$ is obtained by evaluating the $\bar{x}_{\mathrm{H}}$ PDFs at the values of $\bar{x}_{\mathrm{H} \text { I }}$ produced by Global $21 \mathrm{~cm}$ at $z=5.9,7.08$, and 7.54, for every combination of $\boldsymbol{\theta}_{21}$ parameters.

The quasar constraints on $\bar{x}_{\mathrm{H}}$ from McGreer et al. (2015) and Greig et al. (2017) account for sigthline-to-sightline variance. Specifically, McGreer et al. (2015) conduct a jackknife analysis where $\bar{x}_{\mathrm{H}}$ is estimated repeatedly after removing one quasar at a time from their 22-quasar sample. Greig et al. (2017) estimate this effect by computing the $\bar{x}_{\mathrm{H}}$ PDF for $10^{5}$ sightlines extracted from semi-numerical reionization simulations (Mesinger et al. 2016). Recently, Davies et al. (2018) conducted an independent analysis of $\mathrm{J} 1120+0641$ $(z=7.08)$ that accounts for intrinsic emission uncertainty and sightline variance and obtained the estimate $\bar{x}_{\mathrm{HI}}=$ $0.48 \pm 0.26(68 \%)$, which is consistent with Greig et al. (2017) at $<1 \sigma$. The constraint inferred by Mason et al. (2018) from LBGs is tighter than those from $\mathrm{J} 1120+0641$ by Greig et al. (2017) and Davies et al. (2018) at the same redshift, while consistent at the $\sim 1 \sigma$ level. This provides support for an ongoing reionization at $z \approx 7$. We choose to include both $z \approx 7$ constraints (J1120+0641 and LBGs) in our analysis because they are completely independent and, when combined, are expected to produce a more precise and representative estimate of the average fraction of neutral hydrogen.

The constraint from $\mathrm{J} 1342+0928(z=7.54)$ in Bañados et al. (2018) used in this paper only accounts for uncertainty in the quasar's intrinsic emission and does not incorporate the effect of sightline variance. However, we still treat this constraint as
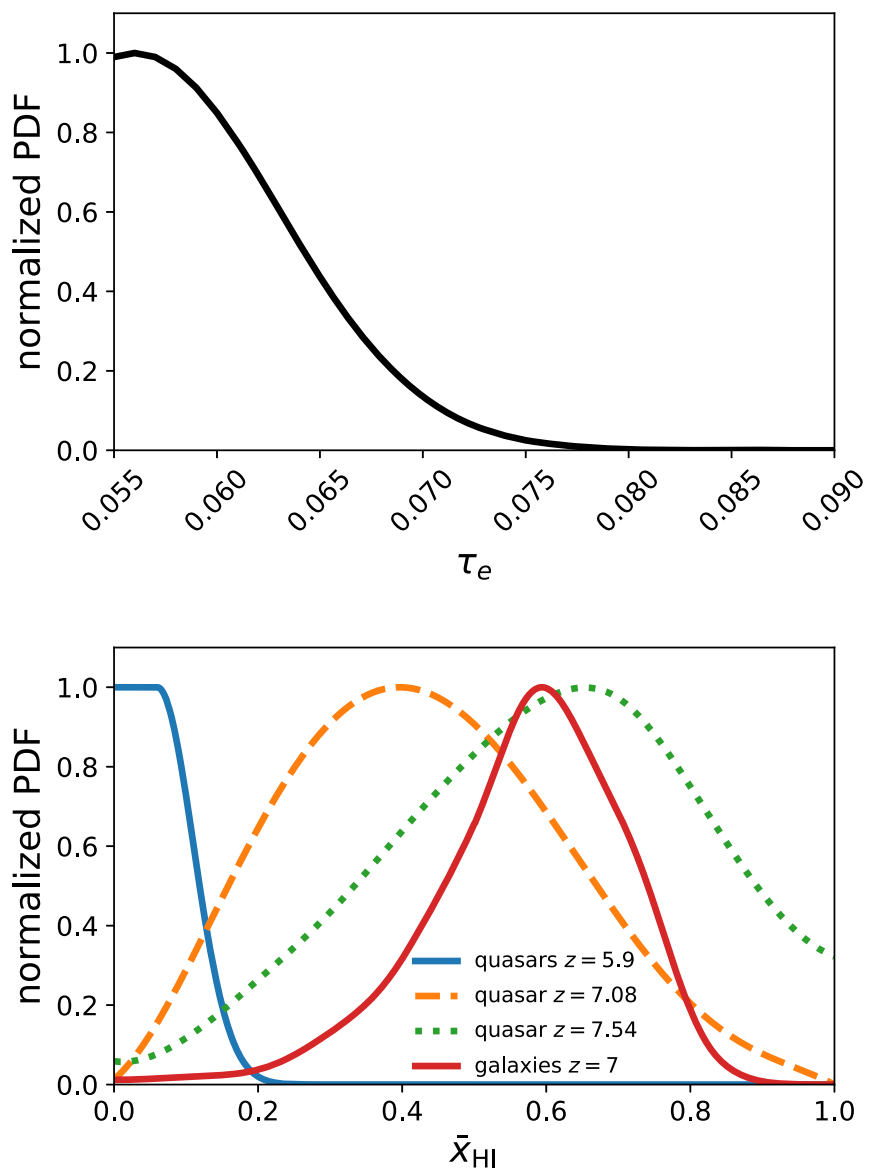

Figure 2. PDFs of the external constraints used in our combined analysis. Each PDF is normalized to its peak amplitude. Top: PDF of the electron scattering optical depth estimated by Planck (Planck Collaboration VI 2018). We model it as a Gaussian centered at $\tau_{\mathrm{e}}=0.056$ and with a width $\sigma=0.007$. Bottom: PDFs of the average neutral hydrogen fraction derived from the spectra of high$z$ quasars and galaxies. The PDF from the quasar at $z=5.9$ was obtained from the fraction of dark $\operatorname{Ly} \alpha$ and $\mathrm{Ly} \beta$ pixels in the quasar spectra (McGreer et al. 2015). The PDFs from the quasars at $z=7.08$ (Greig et al. 2017) and $z=7.54$ (Bañados et al. 2018) were derived from the quasars' Ly $\alpha$ damping wings. The PDF at $z=7$ (Mason et al. 2018) was obtained from the analysis of Ly $\alpha$ transmission from 68 LBGs.

representative at $z=7.54$ because in addition to being their most conservative result, the sightline variance for a significantly neutral IGM is expected to be smaller than for lower neutral hydrogen fractions (McGreer et al. 2011). The same quasar was analyzed by Davies et al. (2018) including the sightline variance effect. They obtained $\bar{x}_{\mathrm{H}}=0.60_{-0.23}^{+0.20}(68 \%)$, consistent with Bañados et al. (2018).

During the preparation of this manuscript, Greig et al. (2019) presented a new analysis of $\mathrm{J} 1342+0928$ that also accounts for the sightline variance effect. Unlike Bañados et al. (2018) and Davies et al. (2018), they do not find evidence for a significantly neutral IGM at $z=7.54$. Their best estimates for $\bar{x}_{\mathrm{HI}}$-which depend on the reionization morphology assumed-are below 0.3 and consistent with zero at $\lesssim 1 \sigma$. However, because the $\bar{x}_{\mathrm{H}}$ PDFs are wide, these results disagree with Bañados et al. (2018) and Davies et al. (2018) only at $\lesssim 1.5 \sigma$. Here we use the conservative estimate from Bañados et al. (2018) and leave incorporating newer quasar constraints on $\bar{x}_{\mathrm{H} \text { I }}$ for future work. 


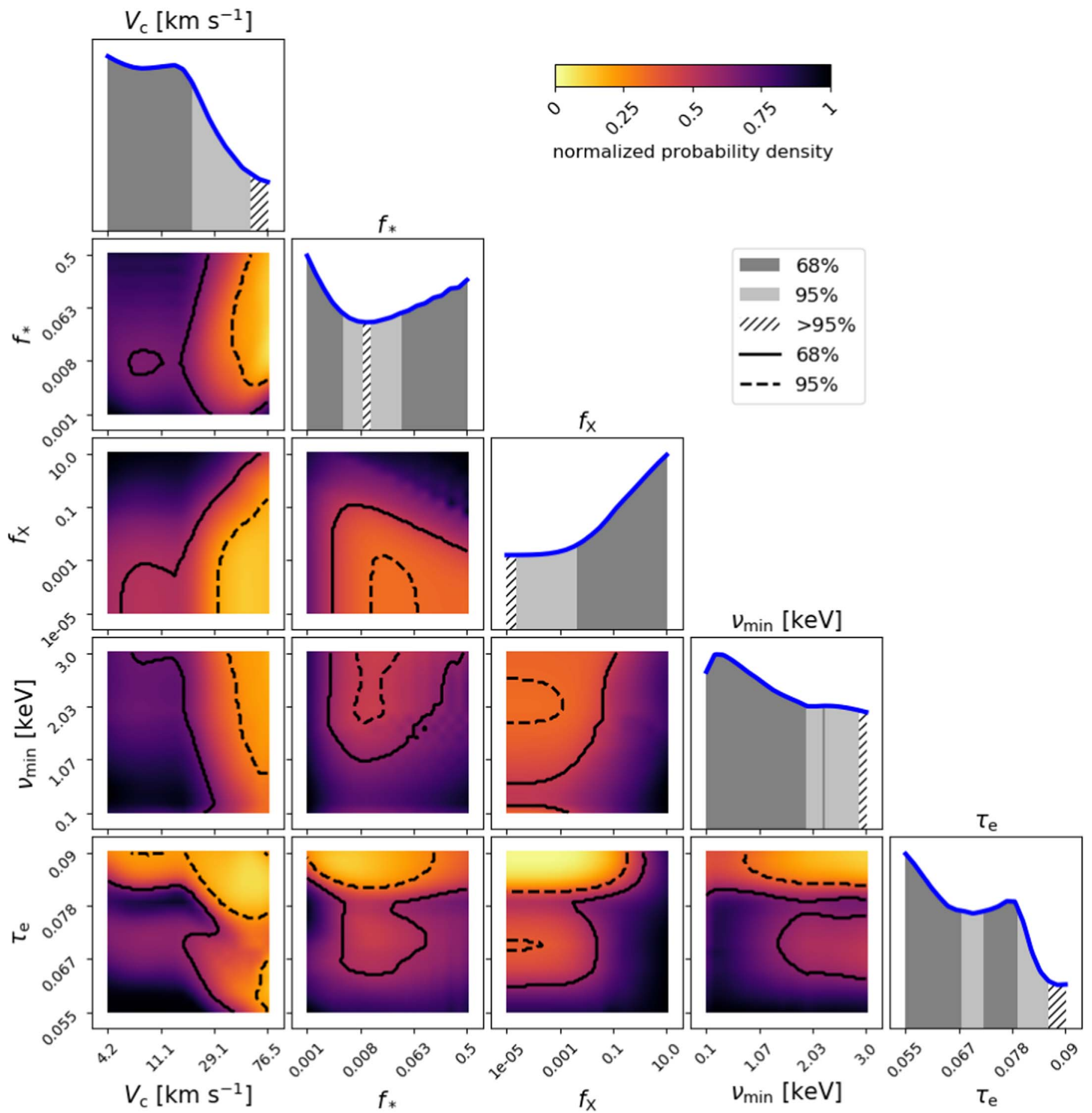

Figure 3. PDFs of the astrophysical parameters derived from the analysis of the EDGES High-Band spectrum alone (Monsalve et al. 2017b) assuming fixed $R_{\mathrm{mfp}}=30 \mathrm{Mpc}$. Each PDF is normalized to its peak amplitude. The regions of parameter space that are disfavored by EDGES (depicted as hatched and light gray bands in the 1D PDFs and as yellow areas on the 2D PDFs) are those of high $V_{\mathrm{c}}$, intermediate $f_{\star}$, low $f_{\mathrm{X}}$, high $\nu_{\min }$, and high $\tau_{\mathrm{e}}$. The marginalized $68 \%$ and $95 \%$ limits obtained from this analysis are listed in Table 2 as case A, as well as in Table 3 for $\tau_{\mathrm{e}}$.

\section{Results}

Now we present the constraints on the six astrophysical parameters derived from the analysis of (1) the EDGES data, (2) the external constraints from Planck+quasars+galaxies, and (3) the combination EDGES+Planck+quasars+galaxies. In particular, we show results for (2) because the external constraints have a significant impact on $V_{\mathrm{c}}$ and $R_{\mathrm{mfp}}$, in addition to $\tau_{\mathrm{e}}$, and it is important to highlight these results independently.
The results for the case where $\nu_{\min }$ is the fifth parameter in the analysis are shown in Figures 3,5, and 6. The results for $R_{\mathrm{mfp}}$ when treated as the fifth parameter are shown in Figure 7. In Figure 7 we do not show the PDFs that only involve the other four parameters because they are similar to those in Figures 3, 5 and 6. Table 2 presents the marginalized $68 \%$ and $95 \%$ limits on all the parameters from the EDGES-only and combined analyses. In Table 3 we show the estimates for $\tau_{\mathrm{e}}$ derived from each individual observation, as well as for different 
Table 2

Marginalized 68\% and 95\% Parameter Limits

\begin{tabular}{|c|c|c|c|c|c|}
\hline \multirow[b]{2}{*}{ Parameter } & \multirow[b]{2}{*}{ Case } & \multicolumn{2}{|c|}{$68 \%$} & \multicolumn{2}{|c|}{$95 \%$} \\
\hline & & Min & Max & Min & Max \\
\hline \multirow{6}{*}{$\overline{V_{\mathrm{c}}\left(\mathrm{km} \mathrm{s}^{-1}\right)}$} & A & 4.2 & 19.3 & 4.2 & 56.0 \\
\hline & B & 4.2 & 21.5 & 4.2 & 58.1 \\
\hline & $\mathrm{C}$ & 6.0 & 11.1 & 4.2 & 52.0 \\
\hline & & 18.6 & 46.6 & & \\
\hline & D & 6.0 & 11.1 & 4.2 & 52.0 \\
\hline & & 17.9 & 46.6 & & \\
\hline \multirow{9}{*}{$f_{*}$} & A & 0.001 & 0.004 & 0.001 & 0.009 \\
\hline & & 0.039 & 0.5 & 0.012 & 0.5 \\
\hline & B & 0.001 & 0.004 & 0.001 & 0.011 \\
\hline & & 0.036 & 0.5 & 0.015 & 0.5 \\
\hline & $\mathrm{C}$ & 0.001 & 0.007 & 0.001 & 0.014 \\
\hline & & 0.062 & 0.5 & 0.019 & 0.5 \\
\hline & D & 0.001 & 0.009 & 0.001 & 0.38 \\
\hline & & 0.036 & 0.045 & & \\
\hline & & 0.053 & 0.324 & & \\
\hline \multirow[t]{4}{*}{$f_{\mathrm{X}}$} & A & 0.0042 & 10 & $2 \times 10^{-5}$ & 10 \\
\hline & B & 0.0025 & 10 & $2 \times 10^{-5}$ & 10 \\
\hline & $\mathrm{C}$ & 0.0021 & 10 & $2 \times 10^{-5}$ & 10 \\
\hline & $\mathrm{D}$ & 0.0012 & 10 & $2 \times 10^{-5}$ & 10 \\
\hline \multirow{3}{*}{$\nu_{\min }(\mathrm{keV})$} & A & 0.1 & 1.9 & 0.1 & 2.9 \\
\hline & & 2.2 & 2.3 & & \\
\hline & $\mathrm{C}$ & 0.1 & 2.0 & 0.1 & 2.9 \\
\hline \multirow[t]{8}{*}{$\tau_{\mathrm{e}}$} & A & 0.055 & 0.067 & 0.055 & 0.086 \\
\hline & & 0.072 & 0.080 & & \\
\hline & B & 0.055 & 0.072 & 0.055 & 0.087 \\
\hline & & 0.074 & 0.079 & & \\
\hline & $\mathrm{C}$ & 0.055 & 0.057 & 0.055 & 0.065 \\
\hline & & 0.059 & 0.063 & & \\
\hline & D & 0.055 & 0.057 & 0.055 & 0.065 \\
\hline & & 0.059 & 0.063 & & \\
\hline \multirow[t]{3}{*}{$R_{\mathrm{mfp}}(\mathrm{Mpc})$} & B & 10.0 & 36.1 & 10.0 & 39.1 \\
\hline & & & & 41.1 & 50.0 \\
\hline & D & 27.5 & 50.0 & 14.3 & 50.0 \\
\hline
\end{tabular}

Note. (1) Cases: (A) EDGES only, $R_{\mathrm{mfp}}=30 \mathrm{Mpc}$; (B) EDGES only, $\nu_{\text {min }}=0.5 \mathrm{keV}$; (C) combined constraints, $R_{\mathrm{mfp}}=30 \mathrm{Mpc}$; and (D) combined constraints, $\nu_{\min }=0.5 \mathrm{keV}$. (2) For some parameters, a given probability volume $(68 \%$ or $95 \%)$ is contained within two or three disjoint value ranges. These ranges are presented in the table as rows associated with the same "Case" letter.

combinations. Unless stated otherwise, the limits quoted for reference in the rest of this section correspond to the case with $\nu_{\min }$ as fifth parameter.

\subsection{EDGES-only Analysis}

As we can see in the 1D and 2D PDFs of Figure 3 and the top row of Figure 7, the EDGES High-Band measurement provides significant discrimination across the explored parameter space. Monsalve et al. (2017b) showed that the HighBand data are incompatible with global signals that have high amplitude and vary rapidly within the band. For our models and parameterization, this translates into the disfavoring of models with high $V_{\mathrm{c}}$, intermediate $f_{*}$, low $f_{\mathrm{X}}$, and high $\tau_{\mathrm{e}}$. Models with high $\nu_{\text {min }}$ and high $R_{\mathrm{mfp}}$ are also disfavored, although the data are less sensitive to variations in these parameters due to their weaker impact on the global signal.
Table 3

Marginalized $68 \%$ Limits for $\tau_{\mathrm{e}}$

\begin{tabular}{lcc}
\hline \hline Observation & Min & Max \\
\hline quasars $z=5.9$ & 0.068 & 0.090 \\
quasar $z=7.08$ & 0.055 & 0.065 \\
quasar $z=7.54$ & 0.055 & 0.065 \\
galaxies $z=7$ & 0.055 & 0.061 \\
Planck & 0.055 & 0.063 \\
EDGES & 0.055 & 0.067 \\
& 0.072 & 0.080 \\
\hline quasars & 0.057 & 0.067 \\
quasars + galaxies & 0.055 & 0.057 \\
quasars + galaxies + Planck & 0.059 & 0.064 \\
quasars + galaxies + Planck + EDGES & 0.055 & 0.058 \\
& 0.059 & 0.063 \\
& 0.055 & 0.057 \\
\end{tabular}

Note. (1) The $\tau_{\mathrm{e}}$ range explored is $0.055-0.090$. As the combined constraints prefer low $\tau_{\mathrm{e}}$, with high probability at $\approx 0.055$, we plan to extend the range below 0.055 in future versions of Global21 cm. (2) These constraints are for $R_{\mathrm{mfp}}=30 \mathrm{Mpc}$. The largest difference in the $\tau_{\mathrm{e}}$ limits when fixing instead $\nu_{\min }=0.5 \mathrm{keV}$ occur when using EDGES data only. These two results are shown as cases A and B in Table 2. For the other observations or combinations, the difference in the $\tau_{\mathrm{e}}$ limits between $R_{\mathrm{mfp}}=30 \mathrm{Mpc}$ and $\nu_{\min }=0.5 \mathrm{keV}$ is $\lesssim 10^{-3}$. (3) In some cases, the $68 \%$ probability volume is contained within two disjoint value ranges. These ranges are presented as two rows.

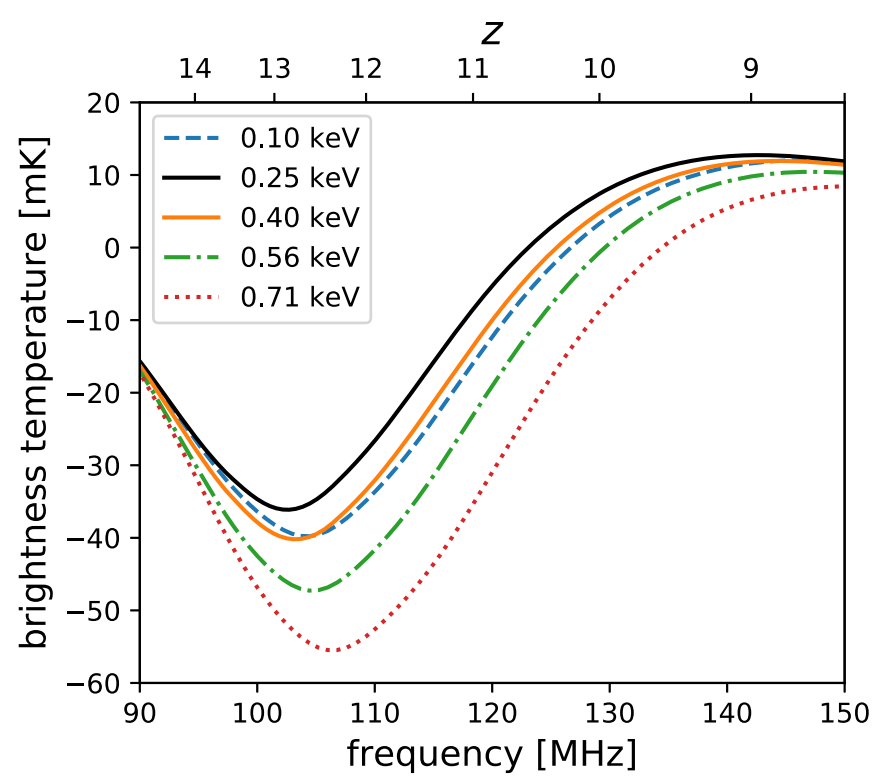

Figure 4. Variation of the global signal for low values of $\nu_{\min }$. Over most of the parameter range explored in this paper, as $\nu_{\min }$ decreases, the absorption amplitude also decreases and the absorption peak is shifted to lower frequencies. However, this trend is reversed below $\nu_{\min } \approx 0.25 \mathrm{keV}$, as shown in this figure. The signals shown here as examples correspond to $V_{\mathrm{c}}=76.5 \mathrm{~km}$ $\mathrm{s}^{-1}, f_{*}=0.026, f_{\mathrm{X}}=10, \tau_{\mathrm{e}}=0.06$, and $R_{\mathrm{mfp}}=31 \mathrm{Mpc}$.

From the PDFs, we derive the following constraints on each one of the parameters:

$V_{\mathrm{c}}$ : Along with $f_{*}, V_{\mathrm{c}}$ determines the timing of the Ly $\alpha$ coupling and drives the evolution of the signal all the way to the onset of heating, affecting the location and depth of the absorption trough. As Cohen et al. (2017) and A. Cohen et al. (2019, in preparation) indicate (e.g., Figure 6 of the latter paper), Ly $\alpha$ coupling is predicted to take place at $z \gtrsim 20$ and therefore cannot be directly probed by EDGES High-Band. 
However, the High-Band data are sensitive to the features of the absorption and this enables us to assign probabilities to the models as a function $V_{\mathrm{c}}$. As we see in the PDFs, high values of $V_{c}$ (i.e., higher minimum mass of star-forming haloes) are disfavored because they result in narrower troughs centered at higher frequencies, to which EDGES has higher sensitivity. We rule out $V_{\mathrm{c}}>19.3 \mathrm{~km} \mathrm{~s}^{-1}$ at $68 \%$ confidence. This velocity threshold is close to the limit of atomic cooling and corresponds to the minimum halo mass of $1.3 \times 10^{8} M_{\odot}$ at $z=10$. At $95 \%$ confidence we rule out $V_{\mathrm{c}}>56 \mathrm{~km} \mathrm{~s}^{-1}$, which corresponds to $M_{\min } \sim 3.1 \times 10^{9} M_{\odot}$ at $z=10$.

$f_{*}$ : Low values of $f_{*}$ result in inefficient Ly $\alpha$ coupling, and as a result, shallow absorption profiles, while high values produce deeper but wider absorption profiles. The EDGES High-Band data and modeling provide low sensitivity to both types of signals, which results in a high probability assigned to low and high $f_{*}$. On the other hand, our analysis disfavors intermediate values of $f_{*}$, which produce sharper signatures in the High-Band range. Specifically, we rule out $0.4 \%<f_{*}<$ $3.9 \%(68 \%)$.

$f_{\mathrm{X}}$ : The X-ray heating efficiency is one of the parameters that control the location of the absorption minimum and the highfrequency slope of the trough. A higher $f_{\mathrm{X}}$ results in sharper but shallower troughs centered at lower frequencies owing to more efficient heating, and could also result in a significant emission feature during reionization. A lower $f_{\mathrm{X}}$ produces deeper and wider troughs centered at higher frequencies, as well as a suppressed or vanishing emission signal. The EDGES spectrum is more sensitive to low- $f_{\mathrm{X}}$ signals, although the high sensitivity expected from their large depth is compensated by the lower sensitivity due to the larger width. As we can see in the PDFs, low values of $f_{\mathrm{X}}$ are disfavored for most of the parameter combinations. After marginalization, we rule out $f_{\mathrm{X}}<0.0042$ $\left(2 \times 10^{-5}\right)$ at $68 \%(95 \%)$ confidence.

$\nu_{\text {min }}$ : Although the global signal is less sensitive to changes in $\nu_{\text {min }}$ than to changes in the previous parameters, this sensitivity is enough for EDGES High-Band to star placing initial constraints. Specifically, EDGES disfavors high values of $\nu_{\mathrm{min}}$, corresponding to harder X-ray SEDs that produce wider and deeper absorption troughs shifted to higher frequencies. As $\nu_{\text {min }}$ decreases, the fraction of soft X-rays emitted by sources increases, which results in more efficient IGM heating and in earlier and shallower absorption troughs. Our conservative $68 \%$ upper limit is $\nu_{\min }=2.3 \mathrm{keV}$; however, as can be seen in the 1D $\nu_{\min }$ PDF and in Table 2, this limit accounts for the narrow range $2.2-2.3 \mathrm{keV}$ that also falls within the $68 \%$ limits. Ignoring this range, we obtain the limit $\nu_{\text {min }}=1.9 \mathrm{keV}(68 \%)$. In the $1 \mathrm{D}$ PDF we also notice that the probability has a peak at $\nu_{\min } \approx 0.25 \mathrm{keV}$ and that it decreases for lower values. We explore the origin of this feature in Figure 4 ; we see that for $\nu_{\min }>0.25 \mathrm{keV}$, and as $\nu_{\min }$ decreases, the absorption trough in the $21 \mathrm{~cm}$ signal becomes shallower and the center is shifted to lower frequencies. However, as values reach and decrease below $\nu_{\min } \approx 0.25 \mathrm{keV}$, the absorption becomes deeper again and the center is shifted to higher frequencies, approaching the shapes observed for $\nu_{\min }>0.25 \mathrm{keV}$. This reversed dependence of the global signal below a $\nu_{\min }$ threshold is due to an effective hardening of the X-ray SED at low $\nu_{\text {min }}$, as most of the energy produced by the sources is deposited very close to the star-forming regions (see Section 2.2.5 of Greig \& Mesinger 2017b). The 1D $\nu_{\text {min }}$
PDF reflects that EDGES High-Band has the lowest constraining capability around this threshold.

$\tau_{\mathrm{e}}$ : Changes in $\tau_{\mathrm{e}}$ affect the evolution of the IGM ionized hydrogen fraction. Higher values imply higher ionizing efficiency of sources, which leads to an earlier reionization and a global signal with a shallower but narrower absorption feature and a weaker emission peak. Lower values of $\tau_{\mathrm{e}}$ result in delayed, deeper, and wider troughs, as well as in a potentially stronger emission feature that peaks at higher frequencies. The general trend in the $\tau_{\mathrm{e}}$ PDFs from EDGES is a probability density that decreases for higher $\tau_{\mathrm{e}}$, which is consistent with the preference of low $\tau_{\mathrm{e}}$ by Planck. Our conservative $68 \%$ upper limit, accounting for the highprobability bump centered at $\tau_{\mathrm{e}} \approx 0.076$ (described in the next paragraph), is $\tau_{\mathrm{e}}=0.080$. We note, however, that due to the higher noise at the low end of the spectrum $(\lesssim 110 \mathrm{MHz})$, the sensitivity of the EDGES High-Band data to models with $\tau_{\mathrm{e}} \gtrsim 0.09$ decreases significantly. Higher-sensitivity measurements at $\lesssim 110 \mathrm{MHz}$, such as those provided by EDGES LowBand, are required to access these higher optical depths.

Beyond the main trend, in Figures 3 and 7 (top row) we see that the $\tau_{\mathrm{e}}$ PDFs have the most irregular structure among the parameters. When projected onto the 1D $\tau_{\mathrm{e}} \mathrm{PDF}$, this structure is seen as a bump at $\tau_{\mathrm{e}} \approx 0.076$. To understand its origin, we compute the PDFs for simulated EDGES spectra. These spectra are produced starting from the five-term foreground model that best fits the measured spectrum, to which we add noise drawn from the same noise profile as the measurement. We also add ripples that mimic those observed in the measured spectrum above the foreground model (see Figure 4 of Monsalve et al. 2017b). In some cases we add ripples only within sub-bands of the spectrum in order to evaluate their specific effect. We find that the bump at $\tau_{\mathrm{e}} \approx 0.076$ is produced by $21 \mathrm{~cm}$ signals that match ripples in the measured spectrum within the range $\approx 125-145 \mathrm{MHz}$. Simulations without these ripples produce PDFs that decrease smoothly with $\tau_{\mathrm{e}}$, without a bump at $\approx 0.076$. Future reprocessing and modeling of the High-Band data might reveal the origin of the ripples. New measurements with different instruments could be used to revise the PDFs of this parameter. As seen in the 1D PDF of Figure 3 , the bump at $\tau_{\mathrm{e}} \approx 0.076$ represents a second range contained within the $68 \%$ confidence limits, in addition to the larger range at low $\tau_{\mathrm{e}}$. Ignoring the bump and considering only the low $\tau_{\mathrm{e}}$ region, the $68 \%$ limit is $\tau_{\mathrm{e}}=0.067$.

$R_{\mathrm{mfp}}$ : The sensitivity of the global signal to changes in $R_{\mathrm{mfp}}$ is lower than for the other parameters and comparable to that for $\nu_{\text {min }}$. Higher values of $R_{\mathrm{mfp}}$ correspond to a faster growth of ionized bubbles, and thus to a faster reionization process and a sharper end of reionization (Greig \& Mesinger 2017a, 2017b). The 1D $R_{\mathrm{mfp}}$ PDF in the top row of Figure 7 shows that the EDGES spectrum disfavors higher values of $R_{\mathrm{mfp}}$. We rule out $R_{\text {mfp }}>36 \mathrm{Mpc}(68 \%)$ for fixed $\nu_{\min }=0.5 \mathrm{keV}$.

In Table 2 the constraints from the EDGES-only analyses are presented as cases A (for fixed $R_{\mathrm{mfp}}=30 \mathrm{Mpc}$ ) and B (for fixed $\left.\nu_{\min }=0.5 \mathrm{keV}\right)$.

\subsection{External Constraints}

Here we describe the constraints on the astrophysical parameters derived from the $\tau_{\mathrm{e}}$ estimate from Planck, the $\bar{x}_{\mathrm{H} \text { I }}$ estimates from quasars at $z=5.9,7.08$, and 7.54, and the $\bar{x}_{\mathrm{H}}$ estimate from galaxies at $z=7$. These external estimates characterize the evolution of the neutral hydrogen fraction and 


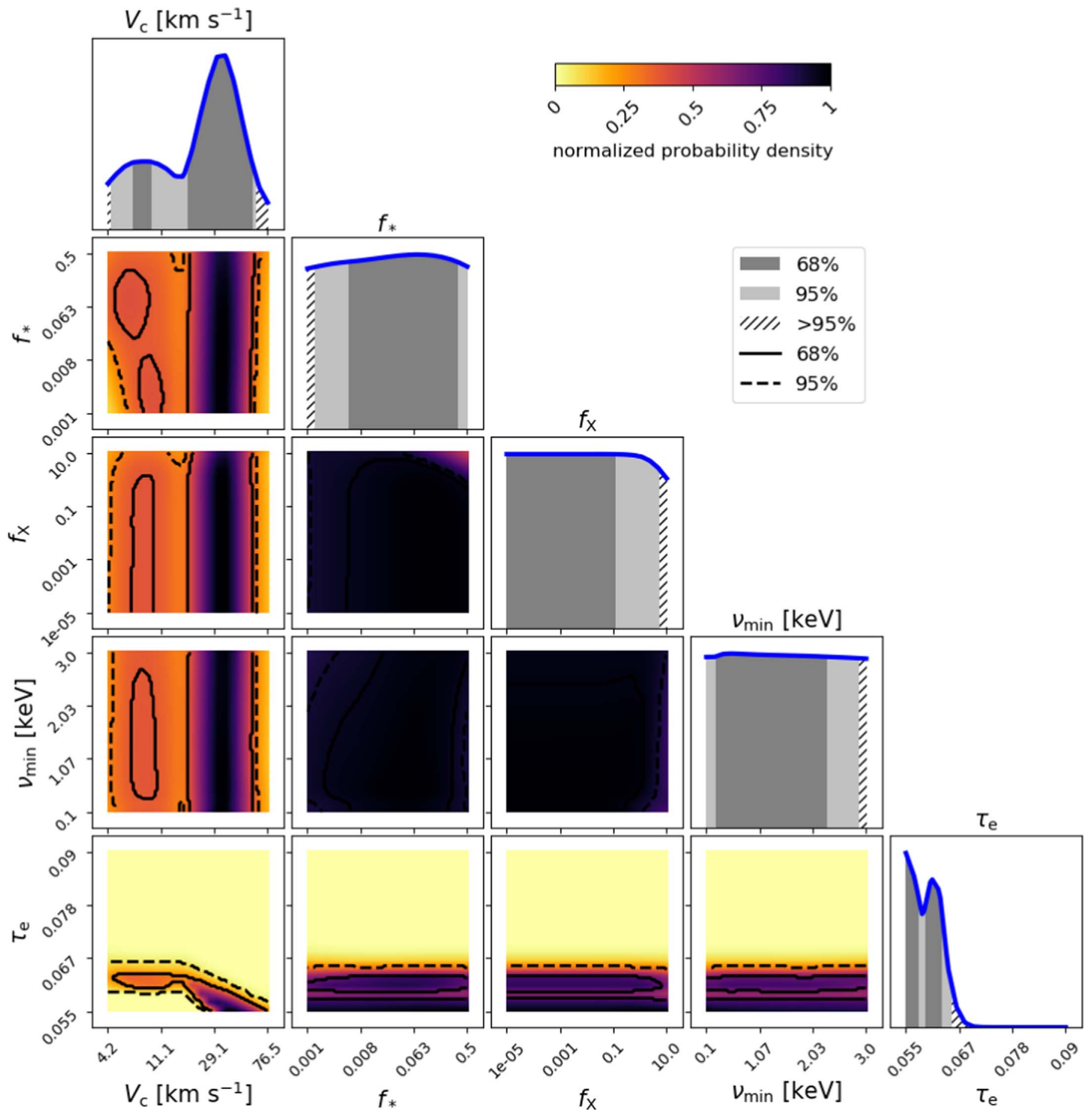

Figure 5. PDFs of the astrophysical parameters derived from the analysis of the external constraints alone and assuming fixed $R_{\mathrm{mfp}}=30 \mathrm{Mpc}$. Each PDF is normalized to its peak amplitude. The external constraints correspond to a prior on $\tau_{\mathrm{e}}$ from Planck (Planck Collaboration VI 2018) and estimates for $\bar{x}_{\mathrm{H}}$ from high- $z$ quasars (McGreer et al. 2015; Greig et al. 2017; Bañados et al. 2018) and LBGs (Mason et al. 2018). The external constraints are described in Section 3.2 and depicted in Figure 2. An important result of this analysis is the strong joint constraint on $\tau_{\mathrm{e}}-V_{\mathrm{c}}$. The marginalized results for $\tau_{\mathrm{e}}$ are summarized in Table 3 .

therefore strongly constrain the reionization parameters, i.e., $\tau_{\mathrm{e}}$ and $R_{\mathrm{mfp}}$. However, due to the correlation between reionization, the star formation history, and-to a lesser degree-heating, there is some degeneracy between the reionization parameters and $V_{\mathrm{c}}$, as well as a much weaker degeneracy with $f_{*}, f_{\mathrm{X}}$, and $\nu_{\text {min }}$. As we show in this section, these degeneracies are reflected in the parameter constraints. The results of the analyses that combine all the external estimates are presented in Figure 5 and in the middle row of Figure 7.
Consider first the limits from the external estimates on $\tau_{\mathrm{e}}$, summarized in Table 3. The upper limit $\bar{x}_{\mathrm{HI}} \leqslant 0.06+0.05$ (68\%) at $z=5.9$ from McGreer et al. (2015) significantly reduces the probability of late reionization and therefore of low $\tau_{\mathrm{e}}$. From this constraint alone we derive the marginalized lower limit $\tau_{\mathrm{e}}>0.068(0.056)$ at $68 \%(99 \%)$ confidence. On the other hand, quasars at $z \gtrsim 7$ suggest that the IGM was significantly neutral at these redshifts, with $\bar{x}_{\mathrm{H}}=0.40_{-0.19}^{+0.21}$ (68\%, Greig et al. 2017) and $\bar{x}_{\mathrm{H}}=0.65_{-0.32}^{+0.15}$ (68\%, Bañados 
et al. 2018) measured at $z=7.08$ and $z=7.54$, respectively. These data complement the upper limit from McGreer et al. (2015) by disfavoring an early reionization and thus high values of $\tau_{\mathrm{e}}$. Specifically, both measurements independently impose the upper limit $\tau_{\mathrm{e}}<0.065(68 \%)$. Finally, the tighter constraint $\bar{x}_{\mathrm{H} \text { I }}=0.59_{-0.15}^{+0.11}(68 \%)$ from LBGs at $z=7$ gives preference to lower optical depths than the quasars. Analyzing this constraint alone results in the upper limit $\tau_{\mathrm{e}}<0.061$ $(68 \%)$, which we note mildly disagrees with the lower limit from McGreer et al. (2015).

Joint analysis of the three quasar constraints favors $\tau_{\mathrm{e}}$ in the range $0.057-0.067(68 \%)$. This range is tighter than current estimates from Planck and in mild disagreement with them ( $\lesssim 2 \sigma$, depending on the specific estimate) because the Planck best fits lie below our quasar 68\% range (Planck Collaboration VI 2018). Combining the quasar and LBG neutral fraction constraints we obtain the upper limit $\tau_{\mathrm{e}}<0.064$ (68\%). Here, the tight neutral fraction constraint from LBGs has increased the consistency between this combined $\tau_{\mathrm{e}}$ result and the $\tau_{\mathrm{e}}$ estimates from Planck. This is noteworthy considering that the assumed ionization histories are different; Planck uses a "tanh" phenomenological dependence of $\bar{x}_{\mathrm{H}}$ on redshift, while we used the realistic neutral fractions produced by Global $21 \mathrm{~cm}$ to derive the quasar and LBG constraints. Finally, incorporating the Planck prior $\left(\tau_{\mathrm{e}}=0.056 \pm 0.007\right)$, we obtain $\tau_{\mathrm{e}}<0.063(68 \%)$. This latter result, derived from the combination of our five external constraints, is the one corresponding to the 1D PDF of Figure 5. In this PDF we see a probability dip in the middle of an otherwise smooth trend of increasing probability toward low values. The dip has the effect of excluding the narrow range $\tau_{\mathrm{e}}=0.058-0.059$ from the $68 \%$ probability region. This feature is explained by the combination of two factors: (1) as we pointed out above, the lower values of $\tau_{\mathrm{e}}$ favored by LBGs (and Planck) are in mild conflict with the higher $\tau_{\mathrm{e}}$ preferred by the quasars; and (2) the piecewise dependence of the SFE on $V_{\mathrm{c}}$, as implemented in Global21cm, which induces features in the PDFs (more details below). Considering that the $\tau_{\mathrm{e}}$ values preferred by the combined constraints are low and reach our current low-end optical depth cutoff even when we include the upper limit on $\bar{x}_{\mathrm{H} \text { I }}$ from McGreer et al. (2015), we plan to extend the parameter range to values below $\tau_{\mathrm{e}}=0.055$ in future versions of Global $21 \mathrm{~cm}$.

The joint constraints on $\tau_{\mathrm{e}}$ and $V_{\mathrm{c}}$ obtained when we apply all the external estimates are shown in the corresponding 2D PDF of Figure 5. This PDF reflects the degeneracy between these two parameters in their effect on the global reionization history. For a fixed $\tau_{\mathrm{e}}$, reionization is slower in the case of low $V_{\mathrm{c}}$. In particular, for low $V_{\mathrm{c}}$ the tail of $\bar{x}_{\mathrm{H}}$ at the end of reionization is longer, and therefore the values of $\bar{x}_{\mathrm{H}_{\mathrm{I}}}$ at a fixed redshift are higher than in the case of higher $V_{\mathrm{c}}$. Hence, for low $\tau_{\mathrm{e}}$ the scenarios with lower $V_{\mathrm{c}}$ are more likely to violate the upper limit on $\bar{x}_{\mathrm{H} \text { I }}$ at $z=5.9$. On the other hand, to keep a sufficiently high $\bar{x}_{\mathrm{H} \text { I }}$ as required at $z \gtrsim 7$, the constraints on the neutral fraction prefer low $V_{\mathrm{c}}$ at low $\tau_{\mathrm{e}}$. As a result, the highprobability region in the $\tau_{\mathrm{e}}-V_{\mathrm{c}} 2 \mathrm{D} \mathrm{PDF}$ is confined to a narrow band that is mainly produced by the complementary effects of the $\bar{x}_{\mathrm{H}}$ constraint at $z=5.9$ and those at $z \gtrsim 7$. At $V_{\mathrm{c}}<16.5 \mathrm{~km} \mathrm{~s}^{-1}$, the band is centered at $\tau_{\mathrm{e}} \approx 0.064$ and only has a weak dependence on $V_{\mathrm{c}}$. At $V_{\mathrm{c}}=16.5 \mathrm{~km} \mathrm{~s}^{-1}$ the band goes through a knee, and for higher $V_{\mathrm{c}}, \tau_{\mathrm{e}}$ decreases for increasing $V_{\mathrm{c}}$. Because the Planck prior and the $\bar{x}_{\mathrm{H} \text { I }}$ estimate from LBGs prefer lower $\tau_{\mathrm{e}}$, the highest probability along the band occurs for high $V_{\mathrm{c}}$. The sharpness of the knee is not physical; it is an artifact of our models produced by the piecewise SFE of Equation (2), which changes the trend exactly at $V_{\mathrm{c}}=16.5 \mathrm{~km} \mathrm{~s}^{-1}$, corresponding to the atomic cooling threshold. We observe that after marginalization, the knee results in relatively sharp features in the 1D PDFs of $V_{\mathrm{c}}$ and $\tau_{\mathrm{e}}$; specifically, probability dips at $V_{\mathrm{c}} \approx 16.5 \mathrm{~km} \mathrm{~s}^{-1}$ and $\tau_{\mathrm{e}} \approx 0.058$. We plan to improve the $V_{\mathrm{c}}$ transition in future modeling.

In the 1D $V_{\mathrm{c}}$ PDF it is more evident that unlike the EDGES data, the external constraints prefer high values of $V_{c}$. This PDF is dominated by a bump that contains most of the $68 \%$ probability volume and peaks at $V_{\mathrm{c}} \approx 35 \mathrm{~km} \mathrm{~s}^{-1}$. The $68 \%$ lower limit of the bump is $V_{\mathrm{c}}=17.9 \mathrm{~km} \mathrm{~s}^{-1}$, i.e., close to the atomic cooling threshold, and the upper limit is $58.1 \mathrm{~km} \mathrm{~s}^{-1}$, which corresponds to $M_{\min } \sim 3.5 \times 10^{9} M_{\odot}$ at $z=10$.

At fixed $\tau_{\mathrm{e}}$ and $V_{\mathrm{c}}$, the 2D PDFs of $f_{*}, f_{\mathrm{X}}$, and $\nu_{\min }$ are nearly flat, reflecting the small effect of these parameters on $\bar{x}_{\mathrm{H}}$. The contribution of $\mathrm{X}$-rays to reionization is non-negligible, however. This can be appreciated better after marginalization, as a mild preference of the data for high $f_{*}$, low $f_{\mathrm{X}}$, and low $\nu_{\text {min }}$, in their 1D PDFs.

As the middle row of Figure 7 shows, the external constraints favor high values of $R_{\mathrm{mfp}}$, which (like high $V_{\mathrm{c}}$ ) correspond to a faster reionization. This is opposite to the preference by EDGES data and results from the need to simultaneously satisfy the neutral fraction upper limit at $z=5.9$ and the high neutral fraction at $z \gtrsim 7$, as well as produce a low optical depth.

\subsection{Combined Analysis}

Next, we present the astrophysical constraints obtained in the analysis that includes the EDGES High-Band data and the external estimates for the optical depth and the neutral hydrogen fraction. Compared to the results from EDGES alone, the external estimates have the strongest impact on the PDFs of $\tau_{\mathrm{e}}, V_{\mathrm{c}}$, and $R_{\mathrm{mfp}}$, while the PDFs of $f_{*}, f_{\mathrm{X}}$, and $\nu_{\text {min }}$ are mainly determined by EDGES. The results are shown in Figure 6 (for fixed $R_{\mathrm{mfp}}=30 \mathrm{Mpc}$ ) and in the bottom row of Figure 7 (for fixed $\nu_{\min }=0.5 \mathrm{keV}$ ). They are also summarized in Table 2 (as cases C and D) and in Table 3 for $\tau_{\mathrm{e}}$.

Considering the 2D PDF for $\tau_{\mathrm{e}}$ and $V_{\mathrm{c}}$ in Figure 6, we see that the narrow high-probability band at low $\tau_{\mathrm{e}}$, produced by the external constraints and introduced in Section 4.2, remains as the main feature. Compared to the result from the external constraints alone, in the combined analysis the EDGES data have the effect of reducing the probability at high $V_{\mathrm{c}}$. This is seen more clearly in the $1 \mathrm{D} V_{\mathrm{c}} \mathrm{PDF}$, where we also note that EDGES produces higher probabilities at lower $V_{\mathrm{c}}$; in particular, the region of low $V_{\mathrm{c}}$ contained within the $68 \%$ probability volume is wider in the case of the combined constraints. From this PDF, we disfavor at $68 \%$ confidence $V_{\mathrm{c}}<6.0 \mathrm{~km} \mathrm{~s}^{-1}$ and $V_{\mathrm{c}}>46.6 \mathrm{~km} \mathrm{~s}^{-1}$; this corresponds to $M_{\min }<3.9 \times 10^{6} M_{\odot}$ and $M_{\min }>1.8 \times 10^{9} M_{\odot}$ at $z=10$. We note that a range of values around the dip at $V_{\mathrm{c}}=16.5 \mathrm{~km} \mathrm{~s}^{-1}$ (the atomic cooling threshold) is also outside the $68 \%$ probability region. At $95 \%$ confidence we derive the upper limit $V_{\mathrm{c}}=52 \mathrm{~km} \mathrm{~s}^{-1}$, equivalent to $2.5 \times 10^{9} M_{\odot}$ at $z=10$.

The combined constraint on $\tau_{\mathrm{e}}$ is driven by Planck + quasars + galaxies, with EDGES having a marginal contribution. Specifically, when we combine EDGES and the external 


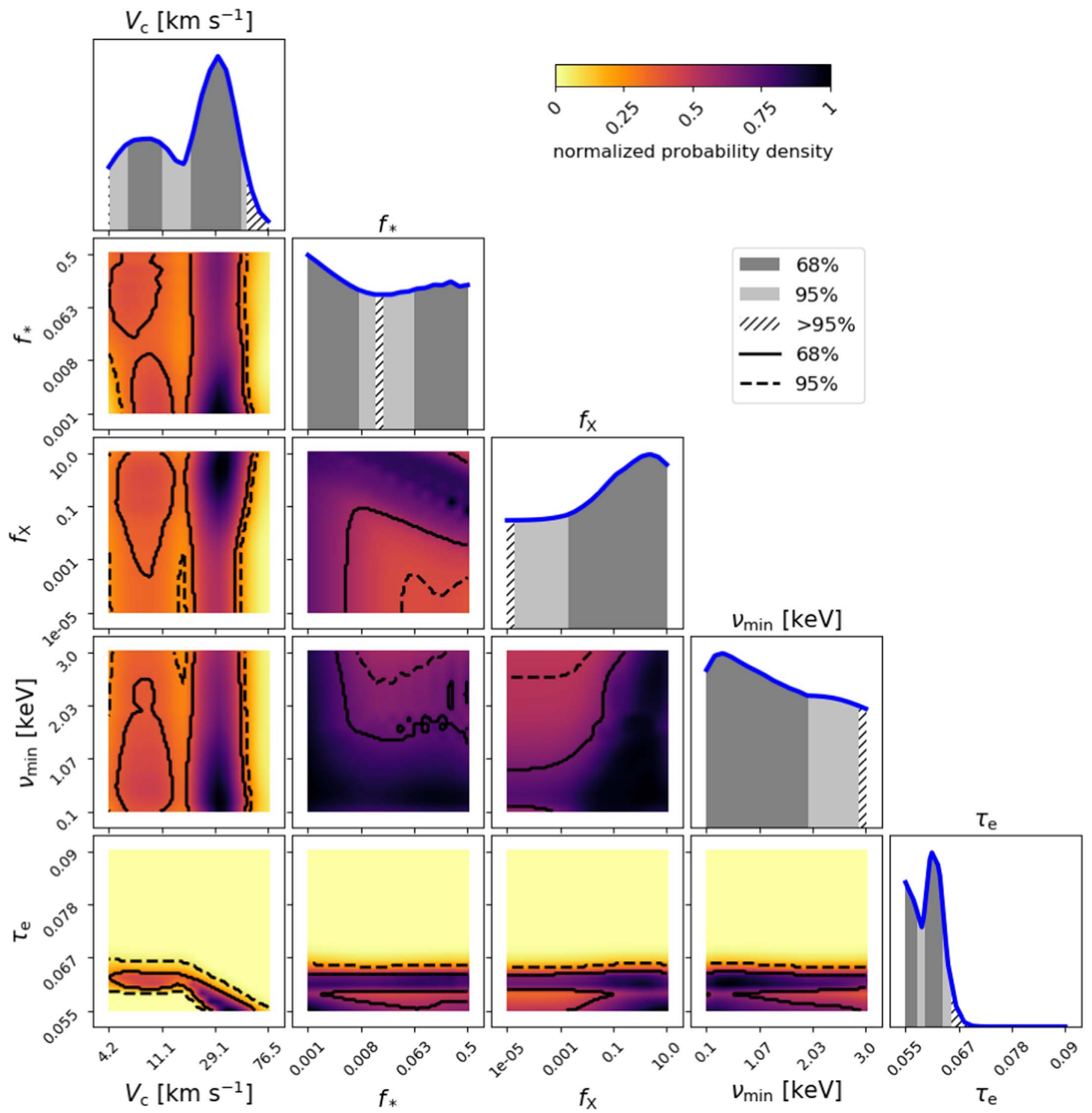

Figure 6. PDFs of the astrophysical parameters derived from the combined analysis that includes the EDGES High-Band spectrum (Monsalve et al. 2017b) and external estimates for $\tau_{\mathrm{e}}$ and $\bar{x}_{\mathrm{H}}$ (McGreer et al. 2015; Greig et al. 2017; Bañados et al. 2018; Mason et al. 2018; Planck Collaboration VI 2018). Here we assume a fixed $R_{\mathrm{mfp}}=30 \mathrm{Mpc}$. Each PDF is normalized to its peak amplitude. Comparing the 1D PDFs in this figure with those in Figures 3 and 5, we see that (1) EDGES drives the constraints on $f_{*}, f_{\mathrm{X}}$, and $\nu_{\min },(2)$ the external observations drive the constraint on $\tau_{\mathrm{e}}$, and (3) EDGES and the external observations impact significantly the constraint on $V_{\mathrm{c}}$. The marginalized $68 \%$ and $95 \%$ limits from this analysis are listed in Table 2 as case $\mathrm{C}$, as well as in Table 3 for $\tau_{\mathrm{e}}$.

observations, we obtain the upper limit $\tau_{\mathrm{e}}<0.063$ at $68 \%$ confidence, with the narrow range $\tau_{\mathrm{e}}=0.057-0.059$ outside the $68 \%$ limits. This is almost identical to the result for Planck + quasars + galaxies alone. It nonetheless reflects a broad consistency between EDGES and the external observations.

The shapes of the $f_{*}, f_{\mathrm{X}}$, and $\nu_{\min }$ 1D PDFs derived from the combined analysis are very close to those found using EDGES alone. As an example of the minor changes in the limits, the
$68 \%$ lower limit on $f_{\mathrm{X}}$ decreases from 0.0042 for EDGES alone to 0.0021 in the combined analysis, which can be explained by the small decrease in the probability of high $f_{\mathrm{X}}$ produced by the external constraints on reionization.

Finally, and as for $\tau_{\mathrm{e}}$, the combined constraint on $R_{\mathrm{mfp}}$ is mainly determined by the external observations. Comparing the 1D PDFs of $R_{\mathrm{mfp}}$ in Figure 7, we see that the PDF derived from the combined analysis (bottom row) is very similar to that 

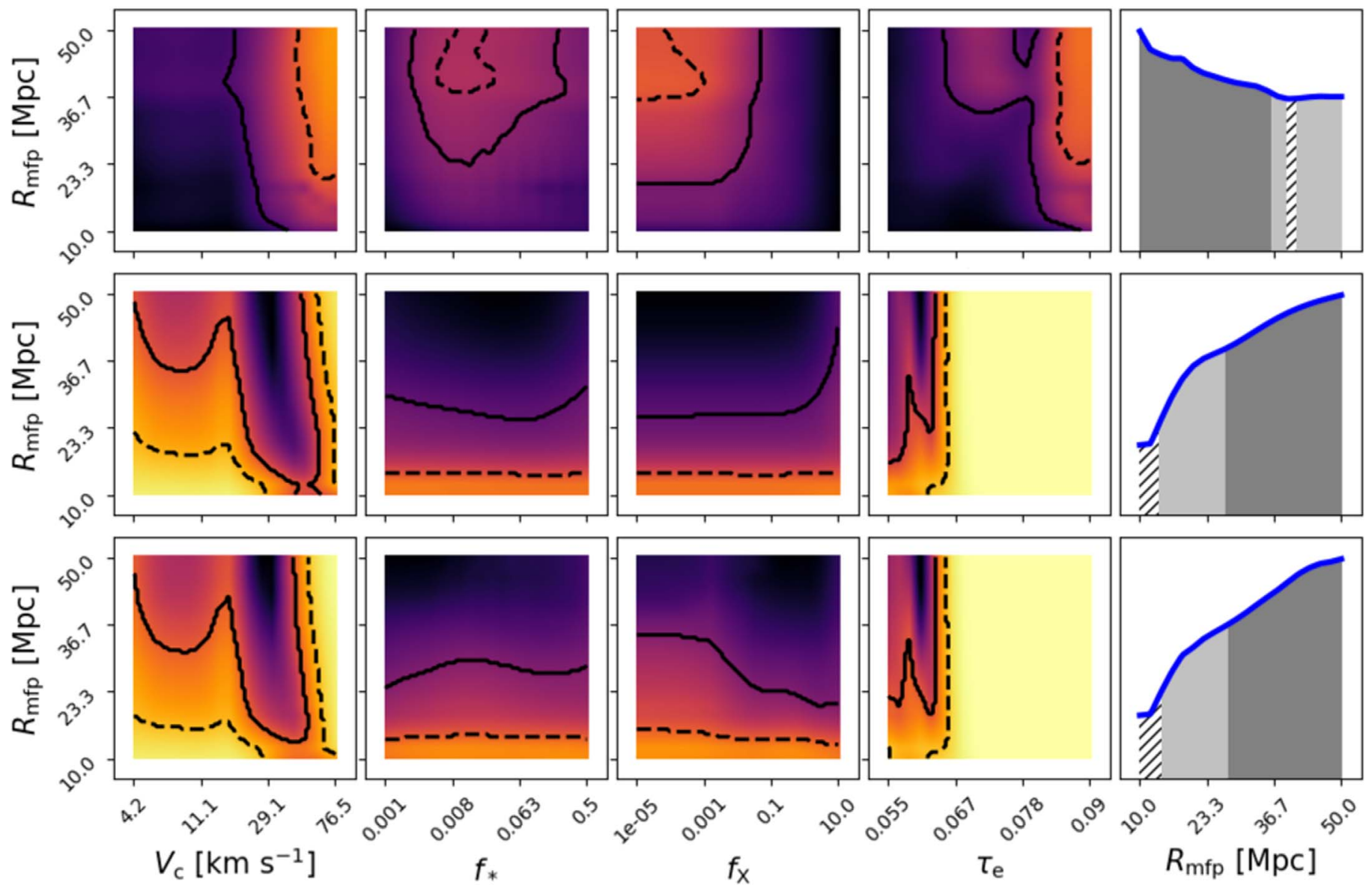

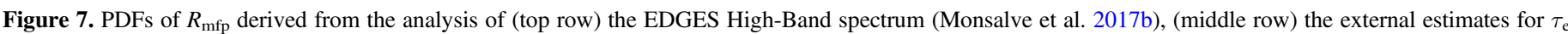

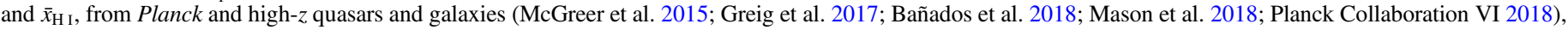

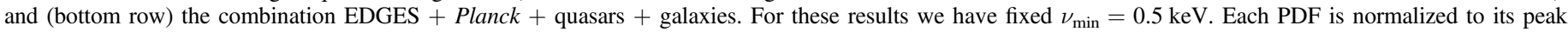

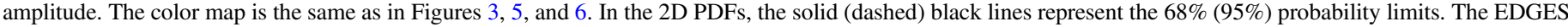

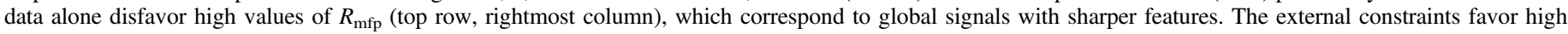

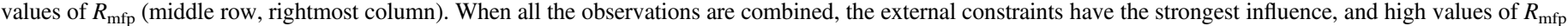
remain preferred (bottom row, rightmost column).

obtained from the external observations alone (middle row). Although the EDGES data disfavor high values of $R_{\mathrm{mfp}}$ (top row), corresponding to sharper global signals, the combined analysis prefers high $R_{\mathrm{mfp}}$ and yields the lower limit $R_{\mathrm{mfp}}>$ $27.5 \mathrm{Mpc}$ at $68 \%$ confidence for fixed $\nu_{\min }=0.5 \mathrm{keV}$.

\section{Discussion}

\subsection{Parameter Degeneracy}

As discussed in Section 4.2, the parameters in our analysis that drive the evolution of the neutral hydrogen fraction are $\tau_{\mathrm{e}}$ and $V_{\mathrm{c}}$, which suffer from a degeneracy in their effect on reionization.

Another example of degeneracy involves the parameters that have an effect on cosmic heating. The global $21 \mathrm{~cm}$ signal is sensitive to the total energy injected into the gas, which depends on several quantities, such as the SFE and the SED of $\mathrm{X}$-rays (including its shape and normalization). In our parameterization, the total number of X-ray photons that contribute to heating is determined by the values of $V_{\mathrm{c}}, f_{*}, f_{\mathrm{X}}$, $\nu_{\min }$, and $\alpha$. As a result, there are clear correlations between these parameters, which manifest themselves in the EDGESonly analysis (Figure 3 ) as diagonal trends on the 2D PDFs of $f_{\mathrm{X}}-V_{\mathrm{c}}, f_{\mathrm{X}}-f_{*}$, (and more weakly) $\nu_{\min }-V_{\mathrm{c}}, \nu_{\min }-f_{*}$, and $\nu_{\min }-f_{\mathrm{X}}$. The correlations demonstrate that via X-ray heating, models with high $f_{\mathrm{X}}$ and high $V_{\mathrm{c}}$ have a signature within the EDGES band that is similar to cases with low $f_{\mathrm{X}}$ and low $V_{\mathrm{c}}$. The diagonal trend on the $f_{\mathrm{X}}-f_{*}$ 2D PDF reflects that in the context of the global signal, the important parameter is $f_{*} f_{\mathrm{X}}$. This degeneracy is broken only for very low values of $f_{*}$, for which Ly $\alpha$ coupling is inefficient. Finally, the degeneracy on the $\nu_{\text {min }}-f_{\mathrm{X}}$ plane reflects that hard spectra (high $\nu_{\text {min }}$ ) generate less heating and require higher $f_{\mathrm{X}}$ to produce absorption troughs similar to models with low values of the two parameters.

The existing degeneracies in the global signal analysis arise because the parameterization of the $3 \mathrm{D}$ simulations used to train Global21cm is not optimized to represent the global signal alone, but instead to track the temporal evolution of the $21 \mathrm{~cm}$ signal within a large cosmological volume from which higher-order statistics, such as power spectra, can also be computed. In the future, an additional tool could be developed to establish consistency between the constraints obtained from radiometric and interferometric measurements, and reduce the degeneracies. Another important remaining task corresponds to finding a set of independent parameters to describe and constrain the astrophysics of the early universe via the global $21 \mathrm{~cm}$ signal alone. We leave this to future work. 


\subsection{Comparison with Previous Results}

In this paper we constrain astrophysical processes during cosmic dawn and reionization, i.e., the same periods that were constrained by Greig \& Mesinger (2017a) and M18. However, as pointed out in Section 1, in these works the astrophysical models were generated using the $21 \mathrm{cmFAST}$ code, which differs from $\mathrm{Global} 121 \mathrm{~cm}$ in details of the processes that are modeled (e.g., Visbal et al. 2012; Fialkov et al. 2013; Cohen et al. 2016). Other differences include different parameterization and parameter ranges explored, as well as the parameters that were kept fixed during the exploration of the parameter space. These differences in modeling prevent us from making a quantitative comparison. The comparison is made even more difficult by the use of different external constraints. Therefore we here limit ourselves to a high-level comparison and leave more detailed discussions for future work.

Greig \& Mesinger (2017a) explored parameters relevant to reionization- $\zeta, T_{\mathrm{vir}}^{\min }$, and $R_{\mathrm{mfp}}$-assuming saturated X-ray heating and a SFE of 5\%. They found that the $\tau_{\mathrm{e}}$ estimate from Planck 2016 and the $\bar{x}_{\mathrm{H}}$ quasar constraints restrict the highprobability range in the joint $\zeta-T_{\mathrm{vir}}^{\min }$ PDF to a relatively narrow band across the plane (see the bottom row of their Figure 8). Although this is a significant result, the band indicates a strong degeneracy between these parameters that prevents tight 1D marginalized constraints, in particular on $\zeta$. In M18 we found that combining the EDGES High-Band data with the Planck 2016 + quasar constraints slightly decreased the degeneracy between $T_{\mathrm{vir}}^{\min }$ and $\zeta$ by reducing the probability of high $T_{\mathrm{vir}}^{\min }$ and high $\zeta$. We also incorporated into the analysis the X-ray heating parameters $L_{\mathrm{X}<2 \mathrm{keV}} / \mathrm{SFR}$ and $E_{0}$, originally introduced in Greig \& Mesinger (2017b). The SFE was still kept at 5\%. We obtained the following 68\% marginalized limits: (1) $5<\log _{10}\left(T_{\text {vir }}^{\min } / \mathrm{K}\right)<5.6$, (2) $10<\zeta<148.4$, and (3) $0.62<E_{0} / \mathrm{keV}<1.5$. We also found that the $68 \%$ confidence region of the soft-band $\mathrm{X}$-ray luminosity was restricted to two ranges: $38<\log _{10}\left(L_{\mathrm{X}<2 \mathrm{keV}} / \mathrm{SFR} / \mathrm{erg} \mathrm{yr} \mathrm{s}^{-1} M_{\odot}^{-1}\right)<39$ and $40.8<\log _{10}\left(L_{\mathrm{X}<2 \mathrm{keV}} / \mathrm{SFR} / \mathrm{erg} \mathrm{yr} \mathrm{s}^{-1} M_{\odot}^{-1}\right)<42$.

The most direct comparison between the results of this paper and those in Greig \& Mesinger (2017a) and M18 corresponds to the constraints on $V_{\mathrm{c}}$ reported here and their limits on $T_{\mathrm{vir}}^{\min }$. These two parameters directly depend on the mass of dark matter halos and are related via (Barkana \& Loeb 2001)

$$
V_{\mathrm{c}}=23.4 \sqrt{\left(\frac{0.6}{\mu}\right)\left(\frac{T_{\mathrm{vir}}^{\min }}{1.98 \times 10^{4}}\right)} \mathrm{km} \mathrm{s}^{-1},
$$

where $\mu$ is the mean molecular weight, which varies between 0.59 for a fully ionized and 1.22 for neutral primordial gas. Qualitatively, the shape of the $V_{\mathrm{c}}$ and $T_{\mathrm{vir}}^{\mathrm{min}}$ 1D PDFs is similar: in the EDGES-only case the probability increases toward lower values, while in the combined analysis a peak occurs at intermediate values. Despite this qualitative agreement, quantitatively, the constraints differ due to a number of discrepancies. The low-end cutoff in $\mathrm{M} 18$ is $T_{\mathrm{vir}}^{\mathrm{min}}=10^{4} \mathrm{~K}$, which corresponds to $V_{\mathrm{c}}=16.5 \mathrm{~km} \mathrm{~s}^{-1}$ (assuming $\mu=0.59$ ). This means that molecular cooling halos are not accounted for in M18. Moreover, the large high-end cutoff in M18, $T_{\text {vir }}^{\min }=10^{6} \mathrm{~K}$, weights the probability toward higher values. This upper limit corresponds to $V_{\mathrm{c}}=166 \mathrm{~km} \mathrm{~s}^{-1}$, i.e., $M_{\min } \sim 8.2 \times 10^{10} M_{\odot}$ at $z=10$, while the upper limit in this paper is $76.5 \mathrm{~km} \mathrm{~s}^{-1}$, corresponding to $M_{\min } \sim 8.0 \times 10^{9} M_{\odot}$ at $z=10$. The $95 \%$ combined constraint on $T_{\mathrm{vir}}^{\min }$ derived in M18 implies $29.6<V_{\mathrm{c}}<117.6 \mathrm{~km} \mathrm{~s}^{-1}$, which corresponds to $4.6 \times$ $10^{8}<M_{\min }<2.9 \times 10^{10} M_{\odot}$ at $z=10$; while our analysis here requires $V_{\mathrm{c}}<52 \mathrm{~km} \mathrm{~s}^{-1}$, or $M_{\min }<2.5 \times 10^{9} M_{\odot}$ at $z=10$. Inclusion of small halos with $4.2<V_{\mathrm{c}}<16.5 \mathrm{~km} \mathrm{~s}^{-1}$ in this work prevents us from determining a strong lower limit on $V_{\mathrm{c}}$. We note that abundance matching techniques applied to the observed luminosity function of galaxies at $z>6$ suggest that star-forming halos of $\sim 10^{10} M_{\odot}$ and lower exist at high redshifts, which disfavors values of $T_{\mathrm{vir}}^{\mathrm{min}}$ higher than $10^{5} \mathrm{~K}$ (e.g., Mashian et al. 2016; Mirocha et al. 2017).

A direct comparison between $V_{\mathrm{c}}$ in this work and $T_{\mathrm{vir}}^{\min }$ in M18 is not straightforward because M18 assumed a fixed $f_{*}=5 \%$, while here we vary $f_{*}$ over $0.1 \%-50 \%$. The effect of $f_{*}$ on the PDFs of $V_{\mathrm{c}}$ and $T_{\mathrm{vir}}^{\min }$ is as follows: for $f_{*}$ much lower than 5\% and all the other parameters fixed, $\zeta$ would be lower, making reionization slower and increasing the neutral fraction at a given redshift. This means that we would need to decrease $V_{\mathrm{c}}$ and $T_{\mathrm{vir}}^{\mathrm{min}}$ in order to produce more ionizing photons and compensate for the lower $\zeta$ when fitting to the $\bar{x}_{\mathrm{H}}$ constraint at $z=5.9$. In other words, the $z=5.9$ constraint would prefer lower values of $V_{\mathrm{c}}$ and $T_{\mathrm{vir}}^{\min }$, in broad agreement to what we see in our analysis when compared to M18. Increasing $f_{*}$ while leaving the other parameters fixed leads to a faster reionization, which is more consistent with the constraint at $z=5.9$.

Comparing the constraints on the ionization parameters in Greig \& Mesinger (2017a), M18, and this work is also non-trivial as $\tau_{\mathrm{e}}$, which we choose to constrain instead of $\zeta$, is an integrated quantity. A. Cohen et al. (2019, in preparation) checked that in Global21cm, when the Planck prior on $\tau_{\mathrm{e}}$ is applied, the allowed $\zeta$ is a growing function of $V_{\mathrm{c}}$ for $V_{\mathrm{c}}>16.5 \mathrm{~km} \mathrm{~s}^{-1}$, in agreement with M18. For lower values of $V_{\mathrm{c}}, \zeta$ is nearly constant due to the effect of feedback mechanisms. In Global $121 \mathrm{~cm}, \zeta$ is also a function of the SFE, which here we vary via $f_{*}$ while in M18 it is kept constant. Therefore, here we expect a larger scatter in $\zeta$ at a given $V_{\mathrm{c}}$. Despite the differences in models and analyses, a high-level consistency between Greig \& Mesinger (2017a), M18, and this paper, is observed in the form of the narrow high-probability bands on the $\zeta-T_{\mathrm{vir}}^{\min }$ and $\tau_{\mathrm{e}}-V_{\mathrm{c}} 2 \mathrm{D}$ PDFs, which are obtained when applying the external $\tau_{\mathrm{e}}$ and $\bar{x}_{\mathrm{H} \mathrm{I}}$ constraints. In addition, the middle and bottom rows of our Figure 7, which show a preference for high $R_{\mathrm{mfp}}$ by the external constraints, are in agreement with the equivalent result in Greig \& Mesinger (2017a).

The X-ray parameters from $21 \mathrm{cmFAST}\left(L_{\mathrm{X}<2 \mathrm{keV}} / \mathrm{SFR}, E_{0}\right)$ and Global $21 \mathrm{~cm}\left(f_{\mathrm{X}}, \nu_{\min }\right)$ are not very sensitive to the external $\tau_{\mathrm{e}}$ and $\bar{x}_{\mathrm{H}}$ estimates and therefore remain mainly constrained by the EDGES spectrum. Although in principle the two codes represent the same physical formalism, different assumptions and prior information lead to the exploration of different parameter ranges between M18 and here. Specifically, in M18 we explore scenarios where the IGM is heated by soft $\mathrm{X}$-rays with (1) an X-ray spectral index $\alpha=-1$, (2) $E_{0}$ varying over $0.1-1.5 \mathrm{keV}$, and (3) an X-ray luminosity equivalent to $f_{\mathrm{X}}$ in the range $\sim 3 \times 10^{-2}-3 \times 10^{2}$. In this work we thoroughly probe a wider range of heating scenarios by exploring $f_{\mathrm{X}}$ in the range $10^{-5}-10^{1}$ and $\nu_{\min }$ between 0.1 and $3 \mathrm{keV}$, assuming an $\mathrm{X}$-ray spectral index $\alpha=-1.3$. A rough estimate, leaving aside the difference in the slope, low-energy cutoff, and X-ray energy range that goes into the definition of $L_{\mathrm{X}}\left(E_{0}-10 \mathrm{keV}\right.$ 
in M18 versus $\nu_{\min }-95 \mathrm{keV}$ here) shows that while in our models the combination $f_{*} f_{\mathrm{X}}$ varies between $10^{-8}$ and 5 , an equivalent combination in M18 (with $f_{*}=5 \%$ ) varies between $1.6 \times 10^{-4}$ and 1.6. Although in this paper we do explore soft $\mathrm{X}$-ray scenarios equivalent to those in M18, our broader parameter ranges enable us to probe many more "cold IGM" cases. Moreover, because here we probe models up to higher values of $\nu_{\min }$, and that they extend to higher X-ray energy (out to $95 \mathrm{keV}$ ), on average our X-rays are harder and less efficient at heating than in M18. As a result, the global signals evaluated in this paper have, on average, deeper absorption troughs shifted to higher frequencies to which the EDGES High-Band spectrum is more sensitive. This enables us to derive the lower limits on $f_{\mathrm{X}}$ listed in Table 2.

Another aspect that leads to the differences in the X-ray constraints is the different range of $V_{\mathrm{c}}$ (equivalently, $T_{\mathrm{vir}}^{\mathrm{min}}$ ) explored in this paper $\left(4.2-76.5 \mathrm{~km} \mathrm{~s}^{-1}\right)$ and in M18 (16.5-166 $\left.\mathrm{km} \mathrm{s}^{-1}\right)$. As the clearest example, Figure 2 of M18 shows regions of high probability for $T_{\mathrm{vir}}^{\mathrm{min}} \gtrsim 10^{5.2} \mathrm{~K}$ that occur at low $L_{\mathrm{X}<2 \mathrm{keV}} / \mathrm{SFR}$ and high $E_{0}$. These high-probability regions are mostly outside the parameter space of this paper as the high-end $V_{\mathrm{c}}$ cutoff here is lower. Thus, they are not projected to the marginalized X-ray PDFs of this paper. However, for $T_{\mathrm{vir}}^{\min } \lesssim 10^{5.2} \mathrm{~K}$, the $L_{\mathrm{X}<2 \mathrm{keV}} / \mathrm{SFR}-T_{\mathrm{vir}}^{\min }$ and $E_{0}-T_{\text {vir }}^{\min }$ PDFs in Figure 2 of M18 do resemble the equivalent $f_{\mathrm{X}}-V_{\mathrm{c}}$ and $\nu_{\min }-V_{\mathrm{c}}$ PDFs in Figure 3 of this paper. This suggests that if a similar parameter space were explored, the constraints on parameters from $21 \mathrm{cmFAST}$ and Global21 cm would become more consistent.

\section{Summary}

We report new constraints on high- $z$ astrophysical parameters derived from the EDGES High-Band measurement of the radio spectrum over 90-190 $\mathrm{MHz}$ (Monsalve et al. 2017b). We show that the spectrum is not only sensitive to reionization, i.e., the electron scattering optical depth and mean-free path of ionizing photons, but can also constrain processes of star formation and heating during cosmic dawn. Specifically, we place limits on the minimum circular velocity (equivalent to the minimum mass) of star-forming halos, the SFE, the X-ray efficiency of sources, and the low-energy cutoff of the X-ray SED. The definition and range of the parameters explored here correspond to the parameterization detailed in Cohen et al. (2017). The models were generated using the new Global2 $1 \mathrm{~cm}$ interpolation tool (A. Cohen et al. 2019, in preparation). These models represent traditional physical scenarios and do not include the exotic physics proposed to explain the EDGES Low-Band measurement (Bowman et al. 2018).

We compute the astrophysical parameter constraints within a Bayesian framework. First, we derive the constraints using the EDGES High-Band data alone. In this case the constraints depend on the sensitivity of the measurement-limited by noise and systematic uncertainty-to the spectral features of the $21 \mathrm{~cm}$ signal within the range $90-190 \mathrm{MHz}$, when simultaneously fitting a model that accounts for the foreground contribution. We then recompute the constraints after incorporating into the analysis a prior on the electron scattering optical depth from Planck (Planck Collaboration VI 2018) and estimates for $\bar{x}_{\mathrm{H} \text { I }}$ at $z \gtrsim 5.9$ from quasars (McGreer et al. 2015; Greig et al. 2017; Bañados et al. 2018) and LBGs (Mason et al. 2018).
Using EDGES data alone, and after marginalization over the foreground parameters and the residual astrophysical parameters, we disfavor at $68 \%$ confidence the following parameter ranges assuming a fixed $R_{\mathrm{mfp}}=30 \mathrm{Mpc}$ :

1. High values of the minimum circular velocity of starforming halos, $V_{\mathrm{c}}>19.3 \mathrm{~km} \mathrm{~s}^{-1}$. This value corresponds to a minimum halo mass of $1.3 \times 10^{8} M_{\odot}$ at $z=10$, which reflects that EDGES High-Band data are sensitive enough to constrain star formation in high-mass halos. Lower values of $V_{\mathrm{c}}$ generate $21 \mathrm{~cm}$ signals with absorption troughs at lower frequencies, which could be constrained more efficiently by Low-Band data.

2. Intermediate values of SFE, $0.4 \%<f_{*}<3.9 \%$. Low values of $f_{*}$ produce $21 \mathrm{~cm}$ signals that fall in the HighBand range but have low amplitude, while high values of $f_{*}$ create troughs that are deep but wide. These types of signals cannot be disfavored with our current sensitivity.

3. Low values of the IGM X-ray heating efficiency, $f_{\mathrm{X}}<0.0042$. After exploring a wide dynamical range of cosmic heating, we robustly disfavor a "cold IGM" scenario.

4. High values of the electron scattering optical depth, $\tau_{\mathrm{e}}>0.08$, thus disfavoring early reionization.

5. High values of the X-ray SED low-frequency cutoff, $\nu_{\text {min }}>2.3 \mathrm{keV}$, constraining the X-ray hardness of the early sources.

When fixing $\nu_{\min }=0.5 \mathrm{keV}$, the EDGES-only analysis also disfavors high values of the mean-free path of ionizing photons, $R_{\mathrm{mfp}}>36.1 \mathrm{Mpc}$.

Combining the EDGES High-Band data with the external observations primarily impacts the results for the parameters that most directly characterize the epoch of reionization: $\tau_{\mathrm{e}}$ and $R_{\mathrm{mfp}}$. However, due to the dependence of the reionization history on star formation, the constraint on $\tau_{\mathrm{e}}$ is degenerate with $V_{\mathrm{c}}$, in particular for $V_{\mathrm{c}}>16.5 \mathrm{~km} \mathrm{~s}^{-1}$, i.e., the atomic hydrogen cooling scenario.

In the combined analysis we obtain the optical depth upper limit $\tau_{\mathrm{e}}<0.063$ at $68 \%$ confidence. We find a similar limit, $\tau_{\mathrm{e}}<0.064$, using only the neutral fraction estimates from quasars and LBGs. This reflects a broad agreement between independent observations despite the different models used for the redshift evolution of the neutral fraction. The EDGES contribution to the combined $\tau_{\mathrm{e}}$ constraint is marginal.

For $V_{\mathrm{c}}$, the combined analysis disfavors at $68 \%$ confidence the ranges $V_{\mathrm{c}}<6.0 \mathrm{~km} \mathrm{~s}^{-1}$ and $V_{\mathrm{c}}>46.6 \mathrm{~km} \mathrm{~s}^{-1}$, while at $95 \%$, it rules out $V_{\mathrm{c}}>52.0 \mathrm{~km} \mathrm{~s}^{-1}$. This result indicates that EDGES High-Band + Planck + quasars + galaxies require the existence of halos with a minimum cooling mass below $2.5 \times 10^{9} M_{\odot}$ at $z=10$. Interestingly, this is consistent with the EDGES absorption feature reported in Low-Band data, which requires efficient star formation in halos well below $10^{10} M_{\odot}$ (Mirocha \& Furlanetto 2019).

The combined analysis reverses the shape of the $R_{\mathrm{mfp}}$ PDF relative to the result from EDGES data alone and assigns higher probabilities to higher values of this parameter, i.e., scenarios with faster growth of ionized bubbles and therefore faster reionization. Specifically, at $68 \%$ confidence we obtain the lower limit $R_{\mathrm{mfp}}>27.5 \mathrm{Mpc}$. Faster reionization scenarios are more compatible with the $\bar{x}_{\mathrm{H} \text { I }}$ upper limit at $z=5.9$ from McGreer et al. (2015) combined with the reports of ongoing reionization at $z \gtrsim 7$ and a low optical depth. 
Finally, the external observations do not significantly affect the results for the other astrophysical parameters, $f_{*}, f_{\mathrm{X}}$, and $\nu_{\min }$, which remain mainly constrained by the EDGES HighBand spectrum.

The results of this paper are in broad agreement with the analyses of Greig \& Mesinger (2017a) and Monsalve et al. (2018), which explored astrophysical models generated with the $21 \mathrm{cmFAST}$ code (Mesinger \& Furlanetto 2007; Mesinger et al. 2011). Nonetheless, noticeable differences occur with the results for $V_{\mathrm{c}}$ and the X-ray heating parameters in Monsalve et al. (2018). These discrepancies are primarily due to (1) making $f_{*}$ a free parameter instead of fixing it at $5 \%$ as in Monsalve et al. (2018); (2) exploring a different range for $V_{c}$, which considers star formation in both atomic and molecular cooling halos; and (3) exploring wider ranges for $f_{\mathrm{X}}$ and $\nu_{\min }$, which extend to scenarios of very inefficient heating due to weak or hard X-ray sources.

We leave for future work detailed comparisons with results for models from $21 \mathrm{cmFAST}$, as well as analyses that incorporate measurements from EDGES Low-Band, which should increase the sensitivity to $21 \mathrm{~cm}$ signals whose main features lie below $\sim 100 \mathrm{MHz}$.

We are grateful to Bradley Greig, Eduardo Bañados, and Charlotte Mason for providing us the hydrogen neutral fraction PDFs derived from quasar ULASJ1120+0641, quasar ULASJ1342+0928, and high- $z$ galaxies, respectively. We also thank Bradley Greig, Kohei Inayoshi, Nicholas Kern, Andrei Mesinger, Jordan Mirocha, and the referee for useful comments and discussions. This work was supported by the NSF through research awards for the Experiment to Detect the Global EoR Signature (AST-0905990, AST-1207761, and AST-1609450). R.A.M. was supported by the NASA Solar System Exploration Virtual Institute cooperative agreement 80ARC017M0006, and by the NASA Ames Research Center grant NNX16AF59G. R.A.M. conducted part of this work at the Astrophysics and Cosmology Research Unit, University of KwaZulu-Natal, South Africa. A.F. is supported by the Royal Society University Research Fellowship. For R.B. and A.C., this publication was made possible by the ISF-NSFC joint research program (grant No. 2580/17) and through the support of a grant from the John Templeton Foundation; the opinions expressed in this publication are those of the authors and do not necessarily reflect the views of the John Templeton Foundation. Computations in this paper were run on the Odyssey cluster supported by the FAS Division of Science, Research Computing Group at Harvard University. EDGES is located at the Murchison Radio-astronomy Observatory. We acknowledge the Wajarri Yamatji people as the traditional owners of the Observatory site. We thank CSIRO for providing site infrastructure and support.

Software: Ipython (https://doi.org/10.1109/MCSE.2007. 53), Numpy (https://doi.org/10.1109/MCSE.2011.37), Scipy (https://doi.org/10.5281/zenodo.1036423), Matplotlib (https:// doi.org/10.5281/zenodo.573577), Astropy (The Astropy Collaboration et al. 2013), Healpy (Górski et al. 2005), h5py (https:// doi.org/10.5281/zenodo.877338).

\section{ORCID iDs}

Raul A. Monsalve (10 https://orcid.org/0000-0002-3287-2327 Anastasia Fialkov (1) https://orcid.org/0000-0002-1369-633X Judd D. Bowman (ㄴ) https://orcid.org/0000-0002-8475-2036
Alan E. E. Rogers (1) https://orcid.org/0000-0003-1941-7458

Thomas J. Mozdzen (1) https://orcid.org/0000-0003-4689-4997

Nivedita Mahesh (1) https://orcid.org/0000-0003-2560-8023

\section{References}

Ali, Z. S., Parsons, A. R., Zheng, H., Pober, J. C., et al. 2015, ApJ, 809, 61 Ali, Z. S., Parsons, A. R., Zheng, H., Pober, J. C., et al. 2018, ApJ, 863, 201 Alvarez, M. A., \& Abel, T. 2012, ApJ, 747, 126

Bañados, E., Venemans, B. P., Mazzucchelli, C., et al. 2018, Natur, 553, 473 Barkana, R. 2016, PhR, 645, 1

Barkana, R. 2018, Natur, 555, 71

Barkana, R., \& Loeb, A. 2001, PhR, 349, 125

Barkana, R., Outmezguine, N. J., Redigolo, D., \& Volansky, T. 2018, PhRvD, 98, 103005

Beardsley, A. P., Hazelton, B. J., Sullivan, I. S., et al. 2016, ApJ, 833, 102 Behroozi, P. S., \& Silk, J. 2015, ApJ, 799, 32

Berlin, A., Hooper, D., Krnjaic, G., \& McDermott, S. D. 2018, PhRvL, 121, 011102

Bernardi, G., Zwart, J. T. L., Price, D., et al. 2016, MNRAS, 461, 2847

Bowman, J. D., Rogers, A. E. E., Monsalve, R. A., Mozdzen, T. J., \& Mahesh, N. 2018, Natur, 555, 67

Ceverino, D., Glover, S. C. O., \& Klessen, R. S. 2017, MNRAS, 470, 2791

Cohen, A., Fialkov, A., \& Barkana, R. 2016, MNRAS, 459, L90

Cohen, A., Fialkov, A., Barkana, R., \& Lotem, M. 2017, MNRAS, 472, 1915

Das, A., Mesinger, A., Pallottini, A., Ferrara, A., \& Wise, J. H. 2017, MNRAS, 469, 1166

Davies, F. B., Hennawi, J. F., Bañados, E., et al. 2018, ApJ, 864, 142

Dowell, J., \& Taylor, G. B. 2018, ApJL, 858, L9

Ewall-Wice, A., Chang, T.-C., Lazio, J., et al. 2018, ApJ, 868, 63

Ewall-Wice, A., Dillon, J. S., Hewitt, J. N., et al. 2016, MNRAS, 460, 4320

Feng, C., \& Holder, G. 2018, ApJL, 858, L17

Fialkov, A., \& Barkana, R. 2014, MNRAS, 445, 213

Fialkov, A., Barkana, R., \& Cohen, A. 2018, PhRvL, 121, 011101

Fialkov, A., Barkana, R., Tseliakhovich, D., \& Hirata, C. M. 2012, MNRAS, 424, 1335

Fialkov, A., Barkana, R., Visbal, E., Tseliakhovich, D., \& Hirata, C. M. 2013, MNRAS, 432, 2909

Fialkov, A., Cohen, A., Barkana, R., \& Silk, J. 2017, MNRAS, 464, 3498

Field, G. B. 1958, PIRE, 46, 240

Fixsen, D. J., Kogut, A., Levin, S., et al. 2011, ApJ, 734, 5

Fragos, T., Lehmer, B. D., Naoz, S., Zezas, A., \& Basu-Zych, A. 2013, ApJL, 776, L31

Fraser, S., Hektor, A., Hütsi, G., et al. 2018, PhLB, 785, 159

Furlanetto, S. R., Oh, S. P., \& Briggs, F. H. 2006, PhR, 433, 181

Gehlot, B. K., Mertens, F. G., Koopmans, L. V. E., et al. 2018, arXiv:1809. 06661

Gilfanov, M., Grimm, H.-J., \& Sunyaev, R. 2004, MNRAS, 347, L57

Górski, K. M., Hivon, E., Banday, A. J., et al. 2005, ApJ, 622, 759

Greig, B., \& Mesinger, A. 2017a, MNRAS, 465, 4838

Greig, B., \& Mesinger, A. 2017b, MNRAS, 472, 2651

Greig, B., Mesinger, A., \& Bañados, E. 2019, MNRAS, 484, 5094

Greig, B., Mesinger, A., Haiman, Z., \& Simcoe, R. 2017, MNRAS, 466, 4239

Grimm, H.-J., Gilfanov, M., \& Sunyaev, R. 2003, MNRAS, 339, 793

Hektor, A., Hütsi, G., Marzola, L., et al. 2018, PhRvD, 98, 023503

Houston, N., Li, C., Li, T., Yang, Q., \& Zhang, X. 2018, PhRvL, 121, 111301

Lehmer, B. D., Xue, Y. Q., Brandt, W. N., et al. 2012, ApJ, 752, 46

Ma, X., Hopkins, P. F., Garrison-Kimmel, S., et al. 2018, MNRAS, 478, 1694

Machacek, M. E., Bryan, G. L., \& Abel, T. 2001, ApJ, 548, 509

Mashian, N., Oesch, P. A., \& Loeb, A. 2016, MNRAS, 455, 2101

Mason, C. A., Trenti, M., \& Treu, T. 2015, ApJ, 813, 21

Mason, C. A., Treu, T., Dijkstra, M., et al. 2018, ApJ, 856, 2

McGreer, I. D., Mesinger, A., \& D’Odorico, V. 2015, MNRAS, 447, 499

McGreer, I. D., Mesinger, A., \& Fan, X. 2011, MNRAS, 415, 3237

Mesinger, A. 2010, MNRAS, 407, 1328

Mesinger, A., \& Furlanetto, S. R. 2007, ApJ, 669, 663

Mesinger, A., Furlanetto, S. R., \& Cen, R. 2011, MNRAS, 411, 955

Mesinger, A., Greig, B., \& Sobacchi, E. 2016, MNRAS, 459, 2342

Mineo, S., Gilfanov, M., \& Sunyaev, R. 2012, MNRAS, 419, 2095

Mirabel, I. F., Dijkstra, M., Laurent, P., Loeb, A., \& Pritchard, J. R. 2011, A\&A, 528, 149

Mirocha, J., \& Furlanetto, S. R. 2019, MNRAS, 483, 1980

Mirocha, J., Furlanetto, S. R., \& Sun, G. 2017, MNRAS, 464, 1365

Mirocha, J., Mebane, R. H., Furlanetto, S. R., Singal, K., \& Trinh, D. 2018, MNRAS, 478, 5591 
Monsalve, R. A., Greig, B., Bowman, J. D., et al. 2018, ApJ, 863, 11

Monsalve, R. A., Rogers, A. E. E., Bowman, J. D., \& Mozdzen, T. J. 2017a, ApJ, 835, 49

Monsalve, R. A., Rogers, A. E. E., Bowman, J. D., \& Mozdzen, T. J. 2017b, ApJ, 847, 64

Mortlock, D., Warren, S. J., Venemans, B. P., et al. 2011, Natur, 474, 616

Mozden, T. J., Bowman, J. D., Monsalve, R. A., \& Rogers, A. E. E. 2016, MNRAS, 455, 3890

Muñoz, J. B., Kovetz, E. D., \& Ali-Haïmoud, Y. 2015, PhRvD, 92, 083528

Muñoz, J. B., \& Loeb, A. 2018, Natur, 557, 684

Paciga, G., Albert, J. G., Bandura, K., et al. 2013, MNRAS, 433, 639

Pâris, I., Petitjean, P., Ross, N. P., et al. 2017, A\&A, 597, A79

Patil, A. H., Yatawatta, S., Koopmans, L. V. E., et al. 2017, ApJ, 838, 65

Pentericci, L., Vanzella, E., Fontana, A., et al. 2014, ApJ, 793, 113

Planck Collaboration VI 2018, arXiv:1807.06209

Planck Collaboration XIII 2016, A\&A, 594, A13

Planck Collaboration XVI 2014, A\&A, 571, A16

Planck Collaboration XLVI 2016, A\&A, 596, A107

Planck Collaboration XLVII 2016, A\&A, 596, A108
Pritchard, J. R., \& Loeb, A. 2008, PhRvD, 78, 103511

Read, J. I., Iorio, G., Agertz, O., \& Fraternali, F. 2016, MNRAS, 462, 3628

Sikivie, P. 2019, PDU, 24, 100289

Singh, S., Subrahmanyan, R., Udaya Shankar, N., et al. 2017, ApJL, 845, L12

Singh, S., Subrahmanyan, R., Udaya Shankar, N., et al. 2018, ApJ, 858, 54

Subrahmanyan, R., \& Cowsik, R. 2013, ApJ, 776, 42

Sun, G., \& Furlanetto, S. R. 2016, MNRAS, 460, 417

Tashiro, H., Kadota, K., \& Silk, J. 2014, PhRvD, 90, 083522

The Astropy Collaboration, Robitaille, T. P., Tollerud, E. J., et al. 2013, A\&A, 558, A33

Tozzi, P., Madau, P., Meiksin, A., \& Rees, M. J. 2000, ApJ, 528, 597

Varshalovich, D. A., \& Khersonskii, V. K. 1977, SvAL, 3, 155

Visbal, E., Barkana, R., Fialkov, A., Tseliakhovich, D., \& Hirata, C. M. 2012, Natur, 487, 70

Voytek, T. C., Natarajan, A., Jáuregui García, J. M., Peterson, J. B., \& López-Cruz, O. 2014, ApJL, 782, L9

Wouthuysen, S. A. 1952, AJ, 57, 31

Xu, H., Wise, J. H., Norman, M. L., Ahn, K., \& O’Shea, B. W. 2016, ApJ, 833, 84 